\title{
Simulation-based cheminformatic analysis of organelle-targeted molecules: lysosomotropic monobasic amines
}

\author{
Xinyuan Zhang $\cdot$ Nan Zheng $\cdot$ Gus R. Rosania
}

Received: 20 September 2007/ Accepted: 5 February 2008/Published online: 13 March 2008

(C) The Author(s) 2008

\begin{abstract}
Cell-based molecular transport simulations are being developed to facilitate exploratory cheminformatic analysis of virtual libraries of small drug-like molecules. For this purpose, mathematical models of single cells are built from equations capturing the transport of small molecules across membranes. In turn, physicochemical properties of small molecules can be used as input to simulate intracellular drug distribution, through time. Here, with mathematical equations and biological parameters adjusted so as to mimic a leukocyte in the blood, simulations were performed to analyze steady state, relative accumulation of small molecules in lysosomes, mitochondria, and cytosol of this target cell, in the presence of a homogenous extracellular drug concentration. Similarly, with equations and parameters set to mimic an intestinal epithelial cell, simulations were also performed to analyze steady state, relative distribution and transcellular permeability in this non-target cell, in the presence of an apicalto-basolateral concentration gradient. With a test set of ninety-nine monobasic amines gathered from the scientific literature, simulation results helped analyze relationships between the chemical diversity of these molecules and their intracellular distributions.
\end{abstract}

Keywords Cheminformatics - Lysosomotropic · Cellular pharmacokinetics · Drug transport .

Electronic supplementary material The online version of this article (doi:10.1007/s10822-008-9194-7) contains supplementary material, which is available to authorized users.

X. Zhang $\cdot$ N. Zheng $\cdot$ G. R. Rosania ( $\square)$

Department of Pharmaceutical Sciences, University of Michigan College of Pharmacy, 428 Church Street, Ann Arbor, MI 48109, USA

e-mail: grosania@umich.edu
Small molecule permeability $\cdot$ Subcellular localization . Simulation $\cdot$ Rational drug design

\section{Introduction}

Weakly basic molecules possessing one or more amine groups accumulate in lysosomes and other membranebound acidic organelles because of the well-known ion trapping mechanism [1-3]. Amines generally have a $\mathrm{p} K_{\mathrm{a}}$ value in the physiological $\mathrm{pH}$ range. Accordingly, they exist as a combination of ionized (protonated) and neutral (unprotonated) species. Because the $\mathrm{pH}$ of lysosomes is one or more units lower than the $\mathrm{pH}$ of the cytosol, the relative concentration of neutral and ionized species inside the lysosomes shifts towards the ionized state. Conversely, because the $\mathrm{pH}$ of the cytosol is higher, the relative concentration of neutral and ionized species in the cytosol shifts towards the neutral state. Since charged molecules are less membrane-permeant, the protonated species become trapped inside the membrane-bounded compartments, relative to the neutral species. Within an acidic lysosome, the concentration of the neutral, membranepermeant species is lower than its concentration in the more basic cytosol. This leads to a concentration gradient of the neutral form of the molecule across the lysosomal membrane, further driving the uptake of the neutral species of the molecule into the acidic organelle.

In medicinal chemistry, the ability to modify the chemical structure of small molecules so as to tailor lysosomotropic behavior may be important for decreasing unwanted side effects, as much as it may be important for increasing efficacy. For many monobasic amines that target extracellular domains of cell surface receptors and ion channels, lysosomal accumulation can be considered as a 
secondary effect of the physicochemical properties of the molecule [4-8]. Previously, many monobasic amines have been experimentally analyzed in cell-based assays, in terms of their ability to accumulate in lysosomes [6, 9-12]. In response to ion trapping, cells exposed to monobasic amines swell and become replete with large vacuoles [6, 9, 10, 13-15]. With a phase contrast microscope, swollen lysosomes can be easily discerned and scored. Furthermore, as monobasic amines accumulate in lysosomes, they can increase the $\mathrm{pH}$ of the organelle through a buffering effect, or by shuttling protons out of the lysosome, across the lysosomal membranes [16]. Therefore, such molecules "compete" with each other for lysosomal accumulation, providing another way to assay for lysosomotropic behavior [16, 17]. A third way to assay lysosomotropic behavior is by labeling lysosomes with fluorescent probes (e.g. LysoTracker ${ }^{\circledR}$ dyes) [17]. As lysosomes expand in response to accumulation of lysosomotropic agents, they accumulate increasing amounts of the LysoTracker ${ }^{\circledR}$ dye and the cells become brightly labeled. By virtue of these effects on live cells, many monobasic amines have been positively identified as "lysosomotropic".

Nevertheless, different studies analyzing lysosomotropic monobasic amines have also identified molecules that deviate from expectations. Furthermore, there is a broad range of concentrations at which vacuolation becomes apparent, spanning several orders of magnitude [10, 18-20]. In addition, there are monobasic amines that do not exhibit any vacuolation-inducing behavior [6, 9, 10, 13, 14, 21], and do not compete with the lysosomal uptake of other lysosomotropic probes $[6,16]$, or that are cytotoxic [21]. Most importantly, some lysosomotropic molecules have been reported to accumulate in other organelles, such as mitochondria [22]. Alprenolol, chlorpromazine, fluoxetine, propranolol and diltiazem are some of the FDA approved drugs in this category $[6,16,22,23]$ that have been classified as being both lysosomotropic and mitochondriotropic by different investigators. In addition, certain monobasic amines may accumulate in lysosomes to a much greater extent than ion-trapping mechanisms would predict [20].

These apparent discrepancies in terms of the lysosomotropic behavior prompted us to begin exploring the relationship between the phenotypic effects of monobasic amines, and their subcellular distribution in lysosomes vs. other organelles. We decided to use a cell-based molecular transport simulator [24, 25] to begin exploring the different possible behaviors of monobasic amines inside cells based on the ion trapping mechanism, paying special attention to their accumulation in lysosomes, cytosol and mitochondria. The simulations help assess the entire range of expected variation in intracellular transport behaviors, based solely on the biophysical principles underlying the ion trapping mechanism. In turn, the expected range of transport behaviors can be related to experimental observations of a lysosomotropic test set of molecules obtained from published research articles. Because the ability to optimize the subcellular transport of small molecules could have practical applications in drug development, we also deem it important to analyze the distribution of molecules inside non-target cells mediating drug transport in the presence of a transcellular concentration gradient. In fact, although direct experimental measurement of subcellular concentration in the presence of a transcellular concentration gradient would be difficult, this may be the most relevant condition for drug uptake and transport throughout the different tissues of the body.

\section{Methods}

Modeling cellular pharmacokinetics of target cells in suspension: the T-model

For subcellular compartments delimited by membranes, passive transport of small molecules in and out of these compartments is determined by the interaction of the molecules with the membrane, the concentration gradient of molecules across the membrane, the local microenvironment on either side of the membrane, and the transmembrane electrical potential [24, 25]. Drug-membrane interactions are largely dependent on the physicochemical properties of small molecules (such as $\mathrm{p} K_{\mathrm{a}}$ and lipophilicity) and the environmental condition (such as local $\mathrm{pH}$ values and membrane potentials). Based on the biophysics of membrane transport, mass transport of drug molecules between different organelles in a cell surrounded by a homogeneous extracellular drug concentration has been modeled mathematically by Trapp and Horobin [25] (Fig. 1, left). Accordingly, three coupled ordinary differential equations (Eqs. 1, 2, and 3) describe the concentration change with time in each subcellular/cellular compartment.

$\frac{d C_{c}}{d t}=\frac{A_{c}}{V_{c}} \times J_{o, c}-\frac{A_{m}}{V_{c}} \times J_{c, m}-\frac{A_{l}}{V_{c}} \times J_{c, l}$,

$\frac{d C_{m}}{d t}=\frac{A_{m}}{V_{m}} \times J_{c, m}$,

$\frac{d C_{l}}{d t}=\frac{A_{l}}{V_{l}} \times J_{c, l}$

where $C$ indicates the concentration, $J$ indicates the flux, $A$ and $V$ indicate the membrane surface area and volume respectively. The subscripts $o, c, l$, and $m$ indicate the extracellular compartment, cytosol, lysosomes, and mitochondria respectively. The directions of fluxes are indicated by the orders of the subscripts, e.g. $J_{c, m}$ represents the flux from 

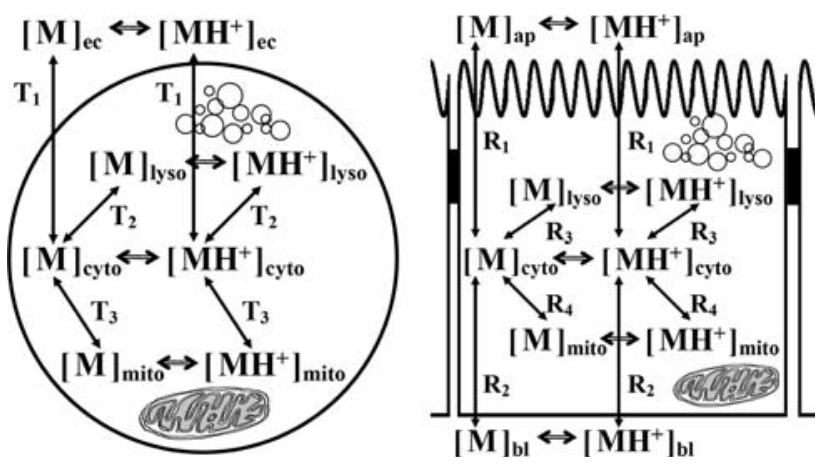

Fig. 1 Diagrams showing the cellular pharmacokinetic phenomena captured by the two mathematical models used in this study: (left) the T-model for a leukocyte-like cell in suspension and (right) the RModel for an epithelia-like cell. Key: ap: apical compartment; bl: basolateral compartment; cyto: cytosol; mito: mitochondria; lyso: lysosome; T1: flux of the ionized/unionized form between the cytosol and the extracellular compartment; T2: flux of the ionized/unionized form between the cytosol and lysosome; T3: flux of the ionized/ unionized form between the cytosol and the mitochondria; R1: flux of the ionized/unionized form between the cytosol and the apical compartment; R2: flux of the ionized/unionized form between the cytosol and the basolateral compartment; R3: flux of the ionized/ unionized form between the cytosol and the lysosome; R4: flux of the ionized/unionized form between the cytosol and the mitochondria

cytosol to mitochondria. Calculations for fluxes between each pair of compartments were the same as described before [25]. The ordinary differential equations were numerically solved (supplemental materials) [24].

An important feature of this model is that at steady state, the drug accumulation in the cytosol is only dependent on the drug concentration outside the cell, the plasma membrane permeability properties, and the ionic conditions of the cytosol and the extracellular medium. Similarly, the drug accumulation inside any given organelle is only dependent on the drug concentration in the cytosol, the permeability properties of the membrane delimiting the organelle, the ionic conditions of the cytosol and the inner lumen of the organelle. Consequently, one can use the same equations to analyze steady state distribution drugs in lysosomes or mitochondria (and other organelles) simply by adjusting the $\mathrm{pH}$ of the organelle, the transmembrane electrical potential, and the organelle volume, surface area, and lipid fraction. For mitochondria, the inner lumen $\mathrm{pH}$ was set at 8 [25] and the membrane potential was set at $-150 \mathrm{mV}$ [26]. Mitochondria were modeled as spheres with $1 \mu \mathrm{m}$ radius. For lysosomes, the inner lumen $\mathrm{pH}$ was set at 5 [1,27-29] and the membrane potential was set at $+10 \mathrm{mV}$ [30]. Leukocytes were modeled as spherical objects of $10 \mu \mathrm{m}$ in diameter. Plasma membrane potential was set at $-60 \mathrm{mV}$ [31]. Extracellular $\mathrm{pH}$ was set at 7.4 (blood). Cytosolic pH was set at 7.0 [32]. Since we are more interested in the drug aqueous concentration in cytosol, the lipid fraction was set at 0 in calculation. Other model parameters were adapted from literature [25]. Hereafter, this cellular pharmacokinetic model applicable to free floating cells in suspension (e.g. leukocytes in circulation) will be dubbed Trapp's Model or 'T-Model'.

Modeling cellular pharmacokinetics of non-target, polarized epithelial cells: the R-model

For modeling drug transport across polarized epithelial cells [24], the cell surface area is divided into apical and basolateral membrane domains (Fig. 1, right). Similarly, the extracellular space is divided into apical and basolateral extracellular compartments. Accordingly, drug uptake into the cell is represented by mass transport of drug molecules from the apical extracellular medium into the cytosol, across the apical membrane. Drug efflux from the cells is represented by mass transport from the cytosol to the basolateral medium, across the basolateral membrane. Because the apical membrane is normally covered with microvilli, the apical membrane surface area $\left(A_{a}\right)$ can be adjusted independently from the basolateral membrane $\left(A_{b}\right)$. Similarly, the extracellular $\mathrm{pH}$ of the apical $\left(p H_{a}\right)$ and basolateral compartments $\left(p H_{b}\right)$, and transmembrane electrical potentials across apical and basolateral membranes $\left(E_{a}\right.$ and $E_{b}$ ) can be independently adjusted, so as to mimic the local microenvironment of the epithelial cells.

A cellular pharmacokinetic model for simulating intracellular concentration and passive transcellular permeability in the presence of a transcellular concentration gradient was developed previously by our group [24,33]. Mass transport across the boundary of each compartment can be described by equations $4-7$.

$\frac{d C_{c}}{d t}=\frac{A_{a}}{V_{c}} J_{a, c}-\frac{A_{m}}{V_{c}} J_{c, m}-\frac{A_{l}}{V_{c}} J_{c, l}-\frac{A_{b}}{V_{c}} J_{c, b}$

$\frac{d C_{m}}{d t}=\frac{A_{m}}{V_{m}} J_{c, m}$,

$\frac{d C_{l}}{d t}=\frac{A_{l}}{V_{l}} J_{c, l}$,

$\frac{d C_{b}}{d t}=\frac{A_{b}}{V_{b}} J_{c, b}$,

The subscripts $a$ and $b$ indicate 'apical' and 'basolateral' respectively. Other symbols and subscripts mean the same as those in the T-model. As in the T-model, the inner lumen $\mathrm{pH}$ of mitochondria was set at 8 [25] and the mitochondrial membrane potential was set at $-150 \mathrm{mV}$ [26]. For lysosomes, the inner lumen $\mathrm{pH}$ was set at 
5 [1, 27-29] and the membrane potential was set at $+10 \mathrm{mV}$ [30]. Epithelial cells were modeled as cubical objects of $10 \mu \mathrm{m}$ in length. Again since we are more interested in the drug aqueous concentration in cytosol the lipid fraction was set to 0 . All other model parameters used in calculation were obtained from the literature [24], and can be found in the supplemental materials. To maintain sink condition in the basolateral compartment, we set the volume of the basolateral compartment $\left(V_{b}\right)$ equal to the human blood volume $(4.7 \mathrm{~L})$.

From simulating cytosol to basolateral flux of molecules in an intestinal epithelial cell, the transcellular permeability of the intestinal epithelial cell monolayer corresponds to the following equation [24]:

$P_{\text {eff }}=\frac{d m_{b}}{C_{a} \cdot A_{a a} \cdot d t}$

where $P_{\text {eff }}$ is the effective permeability, $C_{a}$ is the initial concentration in the apical compartment and is considered to be constant, $d \mathrm{~m}_{\mathrm{b}} / d \mathrm{t}$ is the change in drug mass in the basolateral compartment per unit time, and $A_{a a}$ is the apparent cross sectional area of the cell, which would approximately correspond to the total area of the surface over which drug transport is occurring divided by the number of cells that are effectively transporting drug. Henceforth, this cellular pharmacokinetic model that applies to non-target epithelial cells will be dubbed Rosania's model or 'R-model'.

Analyzing organelle-targeting and transcellular permeability with R- and T-models

To analyze the intracellular distribution of monovalent weakly basic molecules possessing amine functionality, all different combinations of (a) octanol: water partition coefficients of the neutral form of the molecule $\left(\log P_{\mathrm{n}}\right)$; (b) octanol: water partition coefficients of the ionized form of the molecule $\left(\log P_{\mathrm{d}}\right)$; and (c) $\mathrm{p} K_{\mathrm{a}}$ were used as input. $\log P_{\mathrm{n}}$ and $\log P_{\mathrm{d}}$ spanned a range from -5 to +5 , while $\log P_{\mathrm{d}}$ was constrained to a value less than or equal to $\log P_{\mathrm{n}} . \mathrm{p} K_{\mathrm{a}}$ spanned a range from 0 to $14 . \mathrm{p} K_{\mathrm{a}}, \log P_{\mathrm{n}}$, and $\log P_{\mathrm{d}}$ were varied in 0.2 unit increments [24]. The molecular charge $(z)$ was set equal to 1 , which means the simulated whole physicochemical space is specific for monovalent amine-containing molecules. With $\mathrm{R}$ - and T-Model, simulations were performed until the system reached steady state (normally, at $10^{6} \mathrm{~s}$ after beginning of the simulation). For R-Model simulations, the initial apical drug concentration was set at $1 \mathrm{mM}$, and the basolateral drug concentration was set at $0 \mathrm{mM}$. For T-model simulations, extracellular drug concentration was set at $1 \mathrm{mM}$, and kept constant. Accordingly, for each combination of $\mathrm{p} K_{\mathrm{a}}, \log P_{\mathrm{n}}$, and $\log P_{\mathrm{d}}$ used as input, there are seven output values: $\mathrm{C}_{\text {cyto }} \mathrm{R}, \mathrm{C}_{\text {mito }} \mathrm{R}, \mathrm{C}_{\text {lyso }} \mathrm{R}$ (the steady-state cytosolic, mitochondrial and lysosomal concentration estimated with the R-model); $\mathrm{P}_{\text {eff }}$ (the steady-state effective transcellular permeability estimated with the R-Model); and $\mathrm{C}_{\text {cyto }} \mathrm{T}$, $\mathrm{C}_{\text {mito }} \mathrm{T}$, and $\mathrm{C}_{\mathrm{lyso}} \mathrm{T}$ (the steady-state cytosolic, mitochondrial and lysosomal concentrations estimated with the T-Model).

A test set of monobasic amines with associated lysosomotropic behaviors

Focusing on lysosomal targeting, ninety-nine monobasic amines (Table 1) were found by searching PubMed abstracts and titles for articles containing the word "lysosome", "lysosomal", or "lysosomotropic"; from other articles referenced by these articles; and from current review articles describing the lysosomal accumulation of weakly basic molecules [1]. There are more lysosomotropic amine-containing molecules besides molecules included in our table (for example, zwitterions or dibasic amines). However since R- and T-Models have been validated mostly with molecules possessing one ionizable functional group, lysosomotropic amines with more than one ionizable functionality were not included. To estimate the $\mathrm{p} K_{\mathrm{a}}\left(\right.$ at $\left.37^{\circ} \mathrm{C}\right), \log P_{\mathrm{n}}$ and $\log P_{\mathrm{d}}$ for each molecule, we used ChemAxon (http://www.chemaxon.com). A liposomal approximation [24, 44] was applied for $\log P_{\mathrm{n}}$ and $\log P_{\mathrm{d}}$ based on the values obtained from ChemAxon. Intracellular distributions were analyzed for those ninetynine molecules at steady state with the T-model and R-model. Transcellular permeability was analyzed for the ninety-nine molecules at steady state with the R-model.

Interactive visualization of simulation results

Visualization of simulation results was performed with the Miner3D ${ }^{\circledR}$ software package (Dimension 5, Ltd., Slovakia, EU). Simulation results were graphed as $3 \mathrm{D}$ scatter plots to shape the chemical spaces with $\log P_{\mathrm{n}}, \log P_{\mathrm{d}}$ and $\mathrm{p} K_{\mathrm{a}}$ plotted on the three coordinate axes, and the analyzed steady state concentration or permeability determining the color and intensity of the points. For linking simulation results with the test set of lysosomotropic molecules, we used the $\mathrm{p} K_{\mathrm{a}}, \log P_{\mathrm{n}}$ and $\log P_{\mathrm{d}}$ values obtained after liposomal approximations [24].

To plot different chemical spaces we set a threshold concentration value to define accumulation in a specific subcellular compartment. For intracellular concentration, the threshold lysosomal accumulation for lysosomotropic molecules was $\mathrm{C}_{\text {lyso }} \mathrm{T} \geq 2 \mathrm{mM}$ (i.e. two-fold greater than extracellular concentration). The thresholds for selective lysosomal accumulation were $\mathrm{C}_{\text {lyso }} \mathrm{T} \geq 2 \mathrm{mM} ; \mathrm{C}_{\text {lyso }} \mathrm{T} /$ $\mathrm{C}_{\text {mito }} \mathrm{T} \geq 2$; and $\mathrm{C}_{\text {lyso }} \mathrm{T} / \mathrm{C}_{\text {cyto }} \mathrm{T} \geq 2$. The threshold for 
Table 1 The test set of 99 lysosomotropic monobasic amines

\begin{tabular}{|c|c|c|c|c|c|c|c|c|c|c|c|}
\hline Name & $\mathrm{p} K_{\mathrm{a}}$ & $\log P_{\mathrm{n}}$ & $\log P_{\mathrm{d}}$ & $\mathrm{C}_{\text {cyto }} \mathrm{R}$ & $\mathrm{C}_{\text {mito }} \mathrm{R}$ & $\mathrm{C}_{\text {lyso }} \mathrm{R}$ & $\mathrm{P}_{\mathrm{eff}}$ & $\mathrm{C}_{\text {cyto }} \mathrm{T}$ & $\mathrm{C}_{\text {mito }} \mathrm{T}$ & $\mathrm{C}_{\text {lyso }} \mathrm{T}$ & References \\
\hline \multicolumn{7}{|c|}{ Category 1: Low Permeability, Non-lyso, Mito, Non-cyto } & \multicolumn{5}{|c|}{ Chemical space exists } \\
\hline \multicolumn{7}{|c|}{ Category 2: Low Permeability, Non-lyso, Non-mito, Non-cyto } & \multicolumn{5}{|c|}{ Chemical space exists } \\
\hline \multicolumn{7}{|c|}{ Category 3: Low Permeability, Non-lyso, Non-mito, Cyto } & \multicolumn{5}{|c|}{ Chemical space does not exist } \\
\hline \multicolumn{7}{|c|}{ Category 4: Low Permeability, Non-lyso, Mito, Cyto } & \multicolumn{5}{|c|}{ Chemical space exists } \\
\hline \multicolumn{7}{|c|}{ Category 5: Low Permeability, Lyso, Mito, Non-cyto } & \multicolumn{5}{|c|}{ Chemical space exists } \\
\hline \multicolumn{7}{|c|}{ Category 6: Low Permeability, Lyso, Non-mito, Non-cyto } & \multicolumn{5}{|c|}{ Chemical space exists } \\
\hline Lidocaine & 7.2 & 2.71 & 1.16 & 0.15 & 0.06 & 1.74 & 26.67 & 1.87 & 0.81 & 22.26 & {$[10]$} \\
\hline \multicolumn{7}{|c|}{ Category 7: Low Permeability, Lyso, Non-mito, Cyto } & \multicolumn{5}{|c|}{ Chemical space exists } \\
\hline $17-D M A G$ & 7.31 & 2.46 & 0.87 & 0.15 & 0.06 & 1.69 & 13.01 & 2.05 & 0.81 & 22.73 & {$[34]$} \\
\hline Beta-dimethylaminoethylchloride & 7.63 & 2.48 & 0.9 & 0.23 & 0.08 & 1.5 & 11.76 & 2.64 & 0.91 & 17.52 & {$[21]$} \\
\hline Diethylaminoethyl chloride & 8.16 & 2.71 & 1.16 & 0.53 & 0.24 & 1.36 & 19.93 & 3.83 & 1.72 & 9.87 & {$[21]$} \\
\hline Triethanolamine & 8.14 & 1.52 & -0.18 & 0.4 & 0.14 & 1.39 & 0.91 & 3.57 & 1.25 & 12.35 & {$[21]$} \\
\hline \multicolumn{7}{|c|}{ Category 8: Low Permeability, Lyso, Mito, Cyto } & \multicolumn{5}{|c|}{ Chemical space exists } \\
\hline 17-DMAP & 8.3 & 2.47 & 0.89 & 0.62 & 0.31 & 1.35 & 10.79 & 4.17 & 2.08 & 9.07 & {$[34]$} \\
\hline 2-Amino-1-butanol & $\underline{9.49}$ & 2.04 & $\underline{0.55}$ & $\underline{1.67}$ & 9.57 & $\underline{1.29}$ & $\underline{5.84}$ & 10.16 & $\underline{58.1}$ & $\underline{7.82}$ & {$[21]$} \\
\hline 2-Amino-2-methyl-1,3-propanediol & $\underline{9.14}$ & $\underline{1.56}$ & $\underline{0}$ & $\underline{1.44}$ & $\underline{3.32}$ & $\underline{1.3}$ & $\underline{1.58}$ & $\underline{8.01}$ & $\underline{18.52}$ & $\underline{7.22}$ & {$[21]$} \\
\hline$\underline{\text { 2-Amino-2-methyl-1-propanol }}$ & $\underline{9.68}$ & $\underline{1.92}$ & $\underline{0.41}$ & $\underline{1.73}$ & $\underline{14.32}$ & $\underline{1.29}$ & $\underline{4.27}$ & $\underline{10.85}$ & $\underline{89.76}$ & $\underline{8.06}$ & {$[21]$} \\
\hline 2-Aminoethanol(ethanolamine) & $\underline{9.22}$ & $\underline{1.75}$ & $\underline{0.22}$ & $\underline{1.51}$ & $\underline{4.42}$ & $\underline{1.29}$ & $\underline{2.66}$ & $\underline{8.62}$ & 25.18 & $\underline{7.36}$ & {$[21]$} \\
\hline 2-Diethylaminoethanol & $\underline{9.22}$ & $\underline{2.23}$ & $\underline{0.62}$ & $\underline{1.46}$ & $\underline{3.58}$ & $\underline{1.29}$ & $\underline{6.62}$ & $\underline{8.19}$ & 20.09 & $\underline{7.27}$ & {$[21]$} \\
\hline$\underline{\text { 2-Dimethylamino-2-methyl-1-propanol }}$ & $\underline{9.25}$ & $\underline{2.17}$ & $\underline{0.55}$ & $\underline{1.47}$ & $\underline{3.76}$ & $\underline{1.29}$ & $\underline{5.65}$ & $\underline{8.31}$ & 21.23 & $\underline{7.3}$ & {$[21]$} \\
\hline 2-Dimethylaminoethanol & 8.71 & 2.01 & 0.37 & 0.96 & 0.81 & 1.32 & 3.42 & 5.44 & 4.56 & 7.46 & {$[21]$} \\
\hline 2-Methylaminoethanol & $\underline{9.46}$ & $\underline{1.89}$ & $\underline{0.32}$ & $\underline{1.63}$ & 7.29 & $\underline{1.29}$ & $\underline{3.41}$ & $\underline{9.67}$ & $\underline{43.34}$ & $\underline{7.66}$ & {$[21]$} \\
\hline$\underline{\text { 3-Amino-1-propanol }}$ & $\underline{9.49}$ & $\underline{1.77}$ & $\underline{0.24}$ & $\underline{1.66}$ & $\underline{8.67}$ & $\underline{1.29}$ & $\underline{2.85}$ & $\underline{9.99}$ & $\underline{52.26}$ & $\underline{7.76}$ & {$[21]$} \\
\hline 3-Aminopropanal & $\underline{9.14}$ & $\underline{1.77}$ & $\underline{0.24}$ & $\underline{1.46}$ & $\underline{3.6}$ & $\underline{1.29}$ & $\underline{2.76}$ & $\underline{8.17}$ & $\underline{20.17}$ & $\underline{7.25}$ & {$[35]$} \\
\hline 3-Dimethylamino-1-propanol & 8.83 & 2.03 & 0.39 & 1.08 & 1.13 & 1.31 & 3.66 & 5.98 & 6.25 & 7.23 & {$[21]$} \\
\hline 4-Amino-1-butanol & $\underline{9.55}$ & $\underline{1.92}$ & $\underline{0.41}$ & $\underline{1.69}$ & 10.52 & $\underline{1.29}$ & $\underline{4.24}$ & 10.34 & $\underline{64.43}$ & $\underline{7.88}$ & {$[21]$} \\
\hline Ammonia & 8.55 & 1.81 & 0.41 & 1.05 & 1.08 & 1.31 & 3.8 & 5.67 & 5.82 & 7.08 & {$[21]$} \\
\hline Atenolol & $\underline{9.32}$ & $\underline{2.29}$ & $\underline{0.76}$ & $\underline{1.57}$ & $\underline{5.7}$ & $\underline{1.29}$ & $\underline{9.32}$ & $\underline{9.15}$ & $\underline{33.13}$ & $\underline{7.5}$ & {$[6]$} \\
\hline Atropine & $\underline{9.02}$ & $\underline{2.67}$ & $\underline{1.23}$ & $\underline{1.44}$ & $\underline{3.36}$ & $\underline{1.3}$ & 26.87 & $\underline{7.98}$ & $\underline{18.66}$ & $\underline{7.18}$ & {$[10,16]$} \\
\hline Benzylamine & $\underline{9.17}$ & $\underline{2.58}$ & $\underline{1.24}$ & $\underline{1.6}$ & $\underline{6.38}$ & $\underline{1.29}$ & $\underline{28.25}$ & $\underline{9.32}$ & 37.22 & $\underline{7.52}$ & [10] \\
\hline$\underline{\text { Butylamine }}$ & $\underline{9.84}$ & $\underline{2.39}$ & $\underline{0.95}$ & $\underline{1.78}$ & $\underline{24.35}$ & $\underline{1.28}$ & $\underline{14.95}$ & $\underline{11.54}$ & $\underline{157.56}$ & $\underline{8.31}$ & {$[21]$} \\
\hline Diethylamine & $\underline{10.2}$ & $\underline{2.36}$ & $\underline{0.84}$ & $\underline{1.82}$ & $\underline{45}$ & $\underline{1.28}$ & $\underline{11.68}$ & $\underline{12.09}$ & $\underline{298.98}$ & $\underline{8.53}$ & {$[10,21]$} \\
\hline Dimethylamine & $\underline{10.15}$ & $\underline{2.13}$ & $\underline{0.59}$ & $\underline{1.81}$ & $\underline{38.7}$ & $\underline{1.28}$ & $\underline{6.56}$ & $\underline{11.98}$ & $\underline{255.81}$ & $\underline{8.48}$ & {$[21]$} \\
\hline Ethylamine & $\underline{9.86}$ & $\underline{2.11}$ & $\underline{0.62}$ & $\underline{1.78}$ & $\underline{22.73}$ & $\underline{1.28}$ & $\underline{6.99}$ & $\underline{11.46}$ & 146.61 & $\underline{8.28}$ & {$[21]$} \\
\hline Guanidine & $\underline{12.09}$ & $\underline{1.82}$ & $\underline{0.39}$ & $\underline{1.86}$ & $\underline{461.27}$ & $\underline{1.28}$ & $\underline{4.17}$ & $\underline{12.78}$ & $\underline{3164.97}$ & $\underline{8.8}$ & {$[10,21]$} \\
\hline Hexylamine & $\underline{9.84}$ & $\underline{2.66}$ & $\underline{1.24}$ & $\underline{1.79}$ & $\underline{25.48}$ & $\underline{1.28}$ & $\underline{29.17}$ & 11.59 & $\underline{165.24}$ & $\underline{8.33}$ & {$[21]$} \\
\hline Isobutylamine & $\underline{9.87}$ & $\underline{2.4}$ & $\underline{0.95}$ & $\underline{1.79}$ & $\underline{25.47}$ & $\underline{1.28}$ & $\underline{14.96}$ & $\underline{11.59}$ & $\underline{165.19}$ & $\underline{8.33}$ & {$[21]$} \\
\hline$\underline{\text { Isopropanolamine }}$ & $\underline{9.26}$ & $\underline{1.89}$ & $\underline{0.38}$ & $\underline{1.55}$ & $\underline{5.16}$ & $\underline{1.29}$ & $\underline{3.87}$ & $\underline{8.94}$ & $\underline{29.72}$ & $\underline{7.44}$ & {$[21]$} \\
\hline$\underline{\text { Isopropylamine }}$ & $\underline{10.06}$ & $\underline{2.25}$ & $\underline{0.78}$ & $\underline{1.81}$ & $\underline{37.09}$ & $\underline{1.28}$ & $\underline{10.16}$ & $\underline{11.95}$ & $\underline{244.64}$ & $\underline{8.47}$ & {$[21]$} \\
\hline Methylamine & $\underline{9.72}$ & $\underline{2}$ & $\underline{0.5}$ & $\underline{1.74}$ & $\underline{16.1}$ & $\underline{1.29}$ & $\underline{5.27}$ & $\underline{11.02}$ & $\underline{101.71}$ & $\underline{8.12}$ & {$[21]$} \\
\hline Metoclopramide & 8.73 & 2.56 & 0.99 & 1.05 & 1.06 & 1.31 & 14.48 & 5.81 & 5.82 & 7.22 & {$[14,13]$} \\
\hline Morpholine & $\underline{8.21}$ & $\underline{2.02}$ & $\underline{1.25}$ & $\underline{1.36}$ & $\underline{2.95}$ & $\underline{1.3}$ & $\underline{27.55}$ & $\underline{6.62}$ & $\underline{14.36}$ & $\underline{6.31}$ & {$[10]$} \\
\hline $\mathrm{N}$-Acetylprocainamide & 8.73 & 2.51 & 0.93 & 1.04 & 1.02 & 1.31 & 12.59 & 5.76 & 5.66 & 7.25 & {$[10,13]$} \\
\hline NAMA & 8.72 & 2.38 & 0.79 & 1.02 & 0.97 & 1.31 & 9.09 & 5.68 & 5.36 & 7.29 & {$[14]$} \\
\hline N,N-Dimethyl-3-chloropropylamine & 8.38 & 2.5 & 0.92 & 0.69 & 0.38 & 1.34 & 11.66 & 4.41 & 2.45 & 8.54 & {$[21]$} \\
\hline N,N-Dimethyl-benzylamine & 8.67 & 2.84 & 1.3 & 1.02 & 0.98 & 1.31 & 29.42 & 5.65 & 5.4 & 7.25 & {$[10]$} \\
\hline$\underline{\text { Pentylamine }}$ & $\underline{9.84}$ & $\underline{2.53}$ & $\underline{1.09}$ & $\underline{1.78}$ & $\underline{24.35}$ & $\underline{1.28}$ & 20.64 & $\underline{11.54}$ & $\underline{157.56}$ & $\underline{8.31}$ & [21] \\
\hline Practolol & 9.32 & 2.47 & 0.97 & $\underline{1.59}$ & 6.15 & 1.29 & $\underline{15.16}$ & $\underline{9.3}$ & $\underline{35.98}$ & $\underline{7.54}$ & [6] \\
\hline
\end{tabular}


Table 1 continued

\begin{tabular}{|c|c|c|c|c|c|c|c|c|c|c|c|}
\hline Name & $\mathrm{p} K_{\mathrm{a}}$ & $\log P_{\mathrm{n}}$ & $\log P_{\mathrm{d}}$ & $\mathrm{C}_{\text {cyto }} \mathrm{R}$ & $\mathrm{C}_{\text {mito }} \mathrm{R}$ & $\mathrm{C}_{\mathrm{lyso}} \mathrm{R}$ & $\mathrm{P}_{\mathrm{eff}}$ & $\mathrm{C}_{\text {cyto }} \mathrm{T}$ & $\mathrm{C}_{\text {mito }} \mathrm{T}$ & $\mathrm{C}_{\text {lyso }} \mathrm{T}$ & References \\
\hline$\underline{\text { Propylamine }}$ & $\underline{9.85}$ & $\underline{2.27}$ & $\underline{0.8}$ & $\underline{1.78}$ & $\underline{23.26}$ & $\underline{1.28}$ & $\underline{10.58}$ & $\underline{11.49}$ & $\underline{150.19}$ & $\underline{8.29}$ & [21] \\
\hline$\underline{\text { s-Butylamine }}$ & $\underline{10.07}$ & $\underline{2.4}$ & $\underline{0.95}$ & $\underline{1.81}$ & $\underline{39.59}$ & $\underline{1.28}$ & $\underline{15.03}$ & $\underline{12}$ & $\underline{261.77}$ & $\underline{8.49}$ & {$[21]$} \\
\hline$\underline{\text { t-Butylamine }}$ & $\underline{10.27}$ & $\underline{2.27}$ & $\underline{0.81}$ & $\underline{1.83}$ & $\underline{59.14}$ & $\underline{1.28}$ & $\underline{10.92}$ & $\underline{12.26}$ & $\underline{395.97}$ & $\underline{8.59}$ & {$[21]$} \\
\hline$\underline{\text { Triethylamine }}$ & $\underline{9.84}$ & $\underline{2.59}$ & $\underline{1.02}$ & $\underline{1.76}$ & $\underline{18.05}$ & $\underline{1.29}$ & $\underline{17.49}$ & $\underline{11.18}$ & $\underline{114.96}$ & $\underline{8.18}$ & {$[21]$} \\
\hline Trimethylamine & $\underline{9.23}$ & $\underline{2.25}$ & $\underline{0.64}$ & $\underline{1.47}$ & $\underline{3.67}$ & $\underline{1.29}$ & $\underline{6.94}$ & $\underline{8.25}$ & 20.66 & $\underline{7.28}$ & {$[10]$} \\
\hline Tris(hydroxymethyl)methylamine & 8.64 & 1.2 & -0.4 & 0.93 & 0.75 & 1.32 & 0.58 & 5.29 & 4.25 & 7.51 & {$[10,21]$} \\
\hline \multicolumn{7}{|c|}{ Category 9: High Permeability, Non-lyso, Mito, Cyto } & \multicolumn{5}{|c|}{ Chemical space does not exist } \\
\hline \multicolumn{7}{|c|}{ Category 10: High Permeability, Non-lyso, Non-mito, Cyto } & \multicolumn{5}{|c|}{ Chemical space does not exist } \\
\hline \multicolumn{7}{|c|}{ Category 11: High Permeability, Non-lyso, Mito, Non-cyto } & \multicolumn{5}{|c|}{ Chemical space exists } \\
\hline \multicolumn{7}{|c|}{ Category 12: High Permeability, Non-lyso, Non-mito, Non-cyto } & \multicolumn{5}{|c|}{ Chemical space exists } \\
\hline 3-Aminoquinoline & 4.63 & 2.65 & 2.00 & 0.73 & 0.73 & 1.12 & 398.87 & 0.82 & 0.82 & 1.25 & [1] \\
\hline 8-Aminoquinoline & 4.07 & 2.65 & 2.00 & 0.78 & 0.78 & 0.90 & 425.43 & 0.81 & 0.81 & 0.94 & [1] \\
\hline AF-CX1325XX & 1.95 & 2.18 & 0.7 & 0.8 & 0.8 & 0.8 & 148 & 0.81 & 0.81 & 0.81 & [36] \\
\hline Aniline & 4.5 & 2.62 & 1.2 & 0.73 & 0.73 & 1.1 & 372.35 & 0.82 & 0.81 & 1.22 & [10] \\
\hline Benzocaine & 2.7 & 2.78 & 1.41 & 0.8 & 0.8 & 0.8 & 588.46 & 0.81 & 0.81 & 0.82 & [13] \\
\hline Beta-naphthylamine & 4.12 & 2.95 & 1.57 & 0.77 & 0.77 & 0.93 & 838.56 & 0.81 & 0.81 & 0.98 & [10] \\
\hline Pyrimidine & 1.55 & 2.17 & 1.4 & 0.8 & 0.8 & 0.8 & 144.65 & 0.81 & 0.81 & 0.81 & [10] \\
\hline Pyridine & 4.95 & 2.44 & 1.88 & 0.69 & 0.69 & 1.3 & 229.69 & 0.82 & 0.82 & 1.56 & {$[10]$} \\
\hline \multicolumn{7}{|c|}{ Category 13: High Permeability, Lyso, Non-mito, Non-cyto } & \multicolumn{5}{|c|}{ Chemical space exists } \\
\hline 17-AEP & 6.59 & 2.56 & 0.99 & 0.14 & 0.09 & 2.43 & 37.31 & 1.17 & 0.80 & 20.89 & [34] \\
\hline 1-Aminoisoquinoline & 6.88 & 2.74 & 1.94 & 0.36 & 0.30 & 1.45 & 123.44 & 1.53 & 1.28 & 6.16 & {$[1]$} \\
\hline 1-Dodecylimidazole & 6.56 & 3.65 & 3.3 & 0.64 & 0.81 & 1.36 & 2615.12 & 1.27 & 1.61 & 2.7 & [21] \\
\hline Eserine & 6.46 & 3.03 & 1.51 & 0.15 & 0.11 & 2.51 & 137.8 & 1.09 & 0.81 & 17.74 & [10] \\
\hline Harmine & 5.95 & 2.81 & 2.06 & 0.38 & 0.36 & 1.82 & 265.16 & 0.91 & 0.88 & 4.40 & [1] \\
\hline Imidazole & 6.73 & 2.12 & 1.59 & 0.51 & 0.56 & 1.39 & 52.47 & 1.41 & 1.53 & 3.81 & [21] \\
\hline Papaverine & 6.07 & 3.1 & 2.39 & 0.37 & 0.35 & 1.72 & 489.43 & 0.94 & 0.91 & 4.42 & [1] \\
\hline Pilocarpine & 6.39 & 2.38 & 1.89 & 0.48 & 0.51 & 1.44 & 109.54 & 1.1 & 1.18 & 3.32 & [10] \\
\hline s-Collidine & 7.06 & 2.71 & 1.71 & 0.3 & 0.2 & 1.47 & 74.19 & 1.76 & 1.18 & 8.61 & {$[21]$} \\
\hline \multicolumn{7}{|c|}{ Category 14: High Permeability, Lyso, Non-mito, Cyto } & \multicolumn{5}{|c|}{ Chemical space exists } \\
\hline Cyproheptadine & 7.77 & 3.67 & 2.23 & 0.35 & 0.14 & 1.41 & 235.81 & 3.02 & 1.22 & 12.12 & {$[37]$} \\
\hline Diltiazem & 7.89 & 3.08 & 1.57 & 0.37 & 0.14 & 1.4 & 51.38 & 3.23 & 1.25 & 12.07 & {$[16]$} \\
\hline$N$-Dodecylmorpholine & 7.5 & 3.58 & 2.14 & 0.24 & 0.1 & 1.49 & 203.16 & 2.44 & 0.98 & 15.24 & {$[21]$} \\
\hline \multicolumn{7}{|c|}{ Category 15: High Permeability, Lyso, Mito, Non-cyto } & \multicolumn{5}{|c|}{ Chemical space exists } \\
\hline \multicolumn{7}{|c|}{ Category 16: High Permeability, Lyso, Mito, Cyto } & \multicolumn{5}{|c|}{ Chemical space exists } \\
\hline 4-Aminopyridine & $\underline{8.63}$ & $\underline{2.18}$ & $\underline{1.59}$ & $\underline{1.71}$ & $\underline{11.8}$ & $\underline{1.29}$ & $\underline{64.2}$ & $\underline{9.96}$ & $\underline{68.73}$ & $\underline{7.5}$ & {$[10]$} \\
\hline 4-Aminoquinaldine & $\underline{8.5}$ & $\underline{2.7}$ & $\underline{1.82}$ & $\underline{1.49}$ & $\underline{4.21}$ & $\underline{1.29}$ & $\underline{104.97}$ & $\underline{7.87}$ & $\underline{22.31}$ & $\underline{6.85}$ & {$[10]$} \\
\hline 4-Aminoquinoline & 7.98 & 2.65 & 2.00 & 1.29 & 2.56 & 1.30 & 152.07 & 5.73 & 11.40 & 5.79 & {$[1]$} \\
\hline$\underline{\text { 4-Dimethylaminopyridine }}$ & $\underline{8.47}$ & $\underline{2.53}$ & $\underline{1.98}$ & $\underline{1.67}$ & $\underline{9.26}$ & $\underline{1.29}$ & $\underline{156.25}$ & $\underline{9.28}$ & $\underline{51.49}$ & $\underline{7.16}$ & {$[10]$} \\
\hline 9-Aminoacridine & $\underline{8.97}$ & $\underline{3.11}$ & $\underline{2.4}$ & $\underline{1.76}$ & $\underline{18.6}$ & $\underline{1.28}$ & $\underline{419.24}$ & $\underline{10.96}$ & $\underline{115.66}$ & $\underline{7.99}$ & {$[10]$} \\
\hline Alprenolol & $\underline{9.32}$ & $\underline{3.04}$ & $\underline{1.71}$ & $\underline{1.67}$ & $\underline{9.42}$ & $\underline{1.29}$ & $\underline{84.41}$ & 10.09 & $\underline{56.88}$ & $\underline{7.77}$ & {$[6]$} \\
\hline Amantadine & $\underline{10.33}$ & $\underline{2.57}$ & $\underline{2.04}$ & $\underline{1.86}$ & $\underline{288.64}$ & $\underline{1.28}$ & $\underline{186.33}$ & $\underline{12.73}$ & $\underline{1973.95}$ & $\underline{8.77}$ & {$[16]$} \\
\hline Amiodarone & 8.17 & 4.58 & 3.38 & 0.88 & 0.74 & 1.32 & 3439.62 & 4.69 & 3.96 & 7.07 & [4] \\
\hline Amitriptyline & $\underline{9.41}$ & $\underline{3.7}$ & $\underline{2.27}$ & $\underline{1.67}$ & $\underline{9.14}$ & $\underline{1.29}$ & $\underline{306.28}$ & 10.07 & $\underline{55.23}$ & $\underline{7.78}$ & {$[38]$} \\
\hline Biperiden & 8.97 & 3.25 & 1.76 & 1.36 & 2.57 & 1.3 & 89.81 & 7.43 & 14.07 & 7.1 & [39] \\
\hline Chlorphentermine & $\underline{10.24}$ & $\underline{3}$ & $\underline{1.62}$ & $\underline{1.84}$ & $\underline{65.54}$ & $\underline{1.28}$ & $\underline{70.54}$ & $\underline{12.32}$ & $\underline{439.85}$ & $\underline{8.61}$ & {$[40,41]$} \\
\hline Chlorpromazine & 8.87 & 3.7 & 2.27 & 1.33 & 2.33 & 1.3 & 288.89 & 7.19 & 12.66 & 7.05 & [16] \\
\hline$\underline{\text { Desipramine }}$ & $\underline{9.66}$ & $\underline{3.4}$ & $\underline{2.01}$ & $\underline{1.76}$ & $\underline{18.13}$ & $\underline{1.29}$ & $\underline{170.9}$ & $\underline{11.17}$ & $\underline{115.25}$ & $\underline{8.17}$ & {$[12]$} \\
\hline Dibutylamine & 10.36 & 2.93 & 1.48 & 1.84 & 72.43 & 1.28 & 51.13 & 12.37 & 487.37 & 8.63 & [21] \\
\hline
\end{tabular}


Table 1 continued

\begin{tabular}{|c|c|c|c|c|c|c|c|c|c|c|c|}
\hline Name & $\mathrm{p} K_{\mathrm{a}}$ & $\log P_{\mathrm{n}}$ & $\log P_{\mathrm{d}}$ & $\mathrm{C}_{\text {cyto }} \mathrm{R}$ & $\mathrm{C}_{\text {mito }} \mathrm{R}$ & $\mathrm{C}_{\text {lyso }} \mathrm{R}$ & $\mathrm{P}_{\text {eff }}$ & $\mathrm{C}_{\text {cyto }} \mathrm{T}$ & $\mathrm{C}_{\text {mito }} \mathrm{T}$ & $\mathrm{C}_{\text {lyso }} \mathrm{T}$ & References \\
\hline$\underline{\text { Dihydroalprenolol }}$ & $\underline{9.32}$ & $\underline{3.11}$ & $\underline{1.69}$ & $\underline{1.63}$ & $\underline{7.53}$ & $\underline{1.29}$ & $\underline{80.09}$ & $\underline{9.69}$ & $\underline{44.73}$ & $\underline{7.65}$ & [7] \\
\hline Dizocilpine & 8.3 & 3.29 & 1.89 & 0.80 & 0.55 & 1.33 & 110.20 & 4.61 & 3.18 & 7.70 & {$[42]$} \\
\hline$\underline{\text { Dodecylamine }}$ & $\underline{9.84}$ & $\underline{3.44}$ & $\underline{2.12}$ & $\underline{1.8}$ & $\underline{31.89}$ & $\underline{1.28}$ & $\underline{221.84}$ & $\underline{11.8}$ & $\underline{208.84}$ & $\underline{8.41}$ & {$[21]$} \\
\hline Ephedrine & $\underline{9.19}$ & $\underline{2.63}$ & $\underline{1.94}$ & $\underline{1.8}$ & $\underline{31.41}$ & $\underline{1.28}$ & $\underline{146.48}$ & 11.65 & $\underline{202.78}$ & $\underline{8.29}$ & {$[10]$} \\
\hline$\underline{\text { Fluoxetine }}$ & $\underline{9.45}$ & $\underline{3.58}$ & $\underline{3.01}$ & $\underline{1.84}$ & $\underline{69.46}$ & $\underline{1.28}$ & 1731.76 & 12.27 & 463.16 & $\underline{8.55}$ & {$[4,23]$} \\
\hline Imipramine & 8.87 & 3.52 & 2.07 & 1.31 & 2.21 & 1.3 & 181.73 & 7.09 & 11.95 & 7.04 & {$[4]$} \\
\hline Iprindole & $\underline{9.36}$ & $\underline{3.54}$ & $\underline{2.09}$ & $\underline{1.64}$ & $\underline{7.71}$ & $\underline{1.29}$ & $\underline{201.32}$ & $\underline{9.74}$ & $\underline{45.89}$ & $\underline{7.67}$ & [40] \\
\hline Mecamylamine & $\underline{10.49}$ & $\underline{2.93}$ & $\underline{2.27}$ & $\underline{1.86}$ & $\underline{297.05}$ & $\underline{1.28}$ & $\underline{316.44}$ & $\underline{12.73}$ & $\underline{2032.53}$ & $\underline{8.77}$ & {$[10]$} \\
\hline$\underline{\text { Memantine }}$ & $\underline{10.31}$ & $\underline{2.85}$ & $\underline{1.46}$ & $\underline{1.84}$ & $\underline{73.92}$ & $\underline{1.28}$ & $\underline{48.83}$ & $\underline{12.38}$ & $\underline{497.49}$ & $\underline{8.63}$ & [11] \\
\hline Octylamine & $\underline{9.84}$ & $\underline{2.92}$ & $\underline{1.53}$ & $\underline{1.79}$ & $\underline{27.27}$ & $\underline{1.28}$ & $\underline{56.92}$ & $\underline{11.66}$ & $\underline{177.38}$ & $\underline{8.35}$ & [21] \\
\hline Perhexiline & $\underline{10.2}$ & $\underline{3.83}$ & $\underline{3.28}$ & $\underline{1.86}$ & 244.79 & $\underline{1.28}$ & $\underline{3237.19}$ & $\underline{12.7}$ & $\underline{1671.65}$ & $\underline{8.76}$ & {$[4,43]$} \\
\hline$\underline{\text { Phentermine }}$ & $\underline{10.25}$ & $\underline{2.83}$ & $\underline{1.43}$ & $\underline{1.83}$ & $\underline{64.21}$ & $\underline{1.28}$ & $\underline{45.54}$ & $\underline{12.31}$ & $\underline{430.76}$ & $\underline{8.61}$ & {$[40]$} \\
\hline$\underline{\text { Piperidine }}$ & $\underline{10.03}$ & $\underline{2.37}$ & $\underline{1.64}$ & $\underline{1.85}$ & $\underline{148.62}$ & $\underline{1.28}$ & $\underline{74.09}$ & $\underline{12.6}$ & $\underline{1009.79}$ & $\underline{8.71}$ & {$[10]$} \\
\hline Promazine & 8.87 & 3.53 & 2.08 & 1.31 & 2.21 & 1.30 & 185.96 & 7.09 & 11.95 & 7.04 & {$[38]$} \\
\hline Propranolol & $\underline{9.32}$ & $\underline{3.03}$ & $\underline{1.59}$ & $\underline{1.62}$ & $\underline{7.16}$ & $\underline{1.29}$ & $\underline{63.51}$ & $\underline{9.59}$ & $\underline{42.38}$ & $\underline{7.62}$ & {$[10]$} \\
\hline$\underline{\text { Sertraline }}$ & $\underline{9.5}$ & $\underline{3.85}$ & $\underline{2.51}$ & $\underline{1.73}$ & 14.07 & $\underline{1.29}$ & $\underline{537.84}$ & $\underline{10.79}$ & $\underline{87.84}$ & $\underline{8.02}$ & {$[38]$} \\
\hline Thioridazine & 8.61 & 4.01 & 2.61 & 1.11 & 1.27 & 1.31 & 608.81 & 5.96 & 6.80 & 7.02 & {$[38]$} \\
\hline Tributylamine & $\underline{10.44}$ & $\underline{3.45}$ & $\underline{2.1}$ & $\underline{1.85}$ & $\underline{102.49}$ & $\underline{1.28}$ & $\underline{213.44}$ & $\underline{12.51}$ & $\underline{694.01}$ & $\underline{8.68}$ & {$[10]$} \\
\hline Verapamil & 9.33 & 3.7 & 2.27 & $\underline{1.63}$ & $\underline{7.53}$ & 1.29 & $\underline{304.48}$ & $\underline{9.69}$ & 44.71 & $\underline{7.65}$ & [16] \\
\hline
\end{tabular}

Based on simulation results, compounds were classified by permeability $\left(\mathrm{P}_{\text {eff }}\right.$ calculated with the R-model; $\left.\times 10^{-6} \mathrm{~cm} / \mathrm{s} \mathrm{units}\right)$ and subcellular concentrations (calculated with the T-model; $\mathrm{mM}$ units) as follows: Low permeability: $\mathrm{P}_{\mathrm{eff}}<35 \times 10^{-6} \mathrm{~cm} / \mathrm{s}$; High permeability: $\mathrm{P}_{\mathrm{eff}} \geq$ $35 \times 10^{-6} \mathrm{~cm} / \mathrm{s}$; Lyso: $\mathrm{C}_{\text {lyso }} \mathrm{T}>2 \mathrm{mM}$; Mito: $\mathrm{C}_{\text {mito }} \mathrm{T}>2 \mathrm{mM}$; Cyto: $\mathrm{C}_{\text {cyto }} \mathrm{T} \geq 2 \mathrm{mM}$; Non-lyso: $\mathrm{C}_{\text {lyso }} \mathrm{T}<2 \mathrm{mM}$; Non-mito: $\mathrm{C}_{\text {mito }} \mathrm{T}<2 \mathrm{mM}$; Non-cyto: $\mathrm{C}_{\text {cyto }} \mathrm{T}<2 \mathrm{mM}$. Compounds appear in bold if they were reported as non-lysosomotropic in published research articles; in italics if they appear as selective lysosomotropic in the simulations $\left(\mathrm{C}_{\text {lyso }} \mathrm{T} \geq 2 \mathrm{mM} ; \mathrm{C}_{\text {lyso }} \mathrm{T} / \mathrm{C}_{\text {mito }} \mathrm{T} \geq 2 ; \mathrm{C}_{\text {lyso }} \mathrm{T} / \mathrm{C}_{\text {cyto }} \mathrm{T} \geq 2\right)$; underlined if they appear as selectively mitochondriotropic in the simulations $\left(\mathrm{C}_{\text {mito }} \mathrm{T} \geq 2 \mathrm{mM}, \mathrm{C}_{\text {mito }} \mathrm{T} / \mathrm{C}_{\text {lyso }} \mathrm{T} \geq 2, \mathrm{C}_{\mathrm{mito}} \mathrm{T} / \mathrm{C}_{\text {cyto }} \mathrm{T} \geq 2\right)$. In the table, a particular class "exists" if one can find a combination of physicochemical properties (within the range of $\mathrm{p} K_{\mathrm{a}}, \log P_{\mathrm{n}}, \operatorname{and} \log P_{\mathrm{d}}$ input values) that yields the expected behaviour in the simulation

mitochondrial accumulation was $\mathrm{C}_{\text {mito }} \mathrm{T} \geq 2 \mathrm{mM}$. The thresholds for selective mitochondrial accumulation were $\mathrm{C}_{\text {mito }} \mathrm{T} \geq 2 \mathrm{mM} ; \quad \mathrm{C}_{\text {mito }} \mathrm{T} / \mathrm{C}_{\text {lyso }} \mathrm{T} \geq 2 ;$ and $\mathrm{C}_{\text {mito }} \mathrm{T} / \mathrm{C}_{\text {cyto }} \mathrm{T}$ $\geq 2$. The threshold for cytosolic accumulation was $\mathrm{C}_{\text {cyto }} \mathrm{T} \geq 2 \mathrm{mM}$. The thresholds for selective cytosolic accumulation were $\mathrm{C}_{\text {cyto }} \mathrm{T} \geq 2 \mathrm{mM} ; \mathrm{C}_{\text {cyto }} \mathrm{T} / \mathrm{C}_{\text {mito }} \mathrm{T} \geq 2$; and $\mathrm{C}_{\text {cyto }} \mathrm{T} / \mathrm{C}_{\text {mito }} \mathrm{T} \geq 2$. The reason for using the two-fold concentration value as a threshold is because it gave the highest percentage of correct classification and lowest percentage of incorrect classification for the test set of lysosomotropic molecules (as detailed in the Results section).

As recommended by the FDA, the permeability value of metoprolol was used as a threshold to distinguish high vs. low permeability molecules [24]. Previously we calculated permeability for metoprolol, using the $\mathrm{p} K_{\mathrm{a}}$ and $\log P_{n}$ obtained from experimental measurements, to be equal to $35 \times 10^{-6} \mathrm{~cm} / \mathrm{sec}$ [24]. In the present study, we used this value as a threshold to distinguish high vs. low permeability molecules. In addition, we arbitrarily set a value of $1 \times 10^{-6} \mathrm{~cm} / \mathrm{s}$ as a cut-off number to distinguish low from negligible permeability molecules. Accordingly, three permeability classes were defined as: negligible $\left(\mathrm{P}_{\mathrm{eff}}<1\right.$ $\left.\times 10^{-6} \mathrm{~cm} / \mathrm{s}\right)$; low $\left(1 \leq \mathrm{P}_{\mathrm{eff}}<35 \times 10^{-6} \mathrm{~cm} / \mathrm{s}\right)$; and high $\left(\mathrm{P}_{\text {eff }} \geq 35 \times 10^{-6} \mathrm{~cm} / \mathrm{s}\right)$.

\section{Results}

Defining a lysosomal accumulation threshold for lysosomotropic molecules

We began by exploring the simulated property space occupied by monobasic amines, in relation to the test set of molecules obtained from published research articles (Table 1). Three different lysosomal concentration thresholds (2, 4 and $8 \mathrm{mM}$ ) were tested in terms of their ability to discriminate lysosomotropic vs. non-lysosomotropic compounds (Fig. 2). For compounds with $\geq 2 \mathrm{mM}$ accumulation in lysosomes (Fig. 2a-d), eight (8) of the test compounds were below the accumulation threshold (Fig. 2a, b), while ninety-one (91) were above the threshold (Fig. 2c, d). For compounds with $\geq 4 \mathrm{mM}$ accumulation in lysosomes (Fig. 2e-h), twelve (12) of the test compounds 


$$
C_{\text {lyso }} T \geq 2 \mathrm{mM}
$$
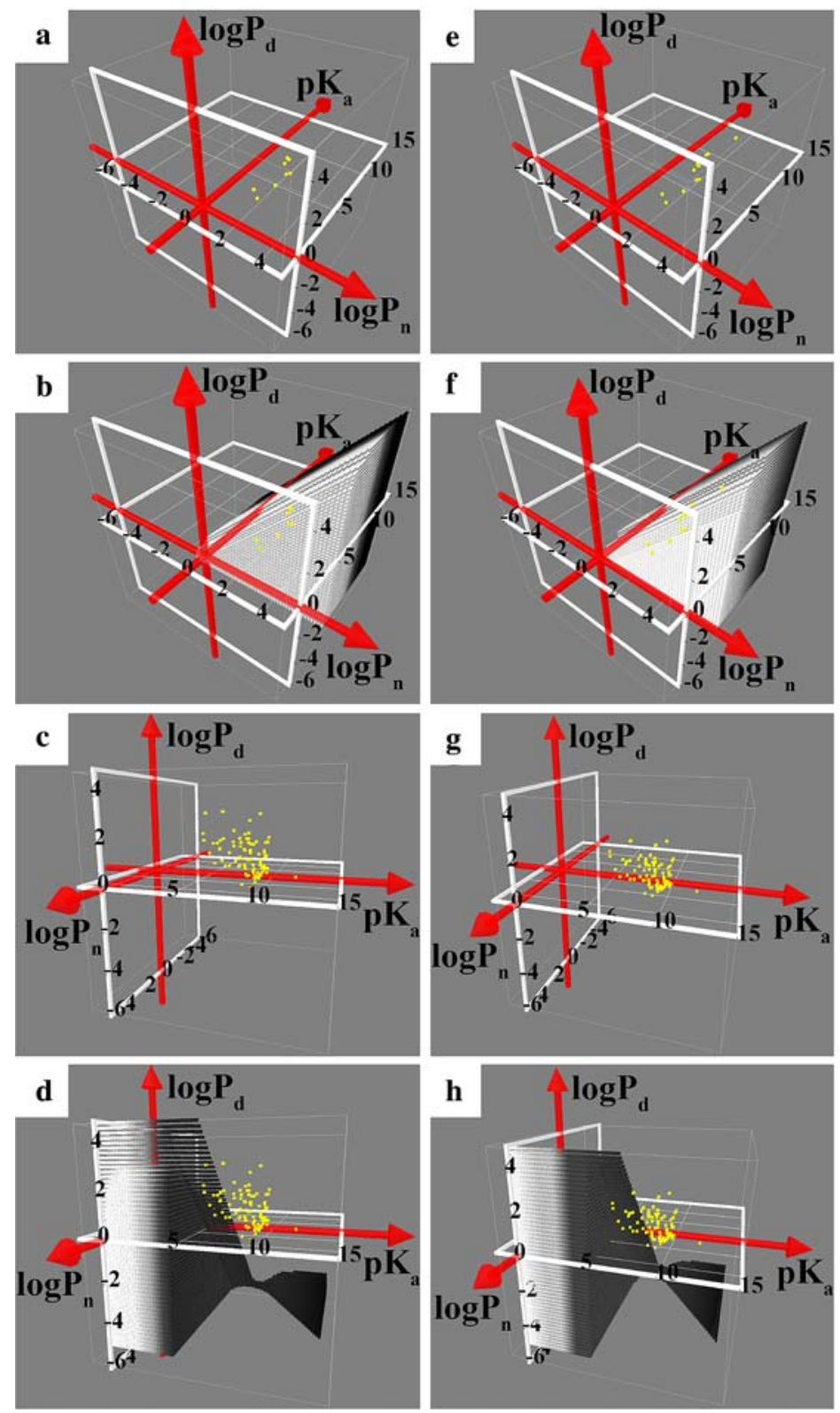

$C_{\text {lyso }} \mathrm{T} \geq 4 \mathrm{mM}$
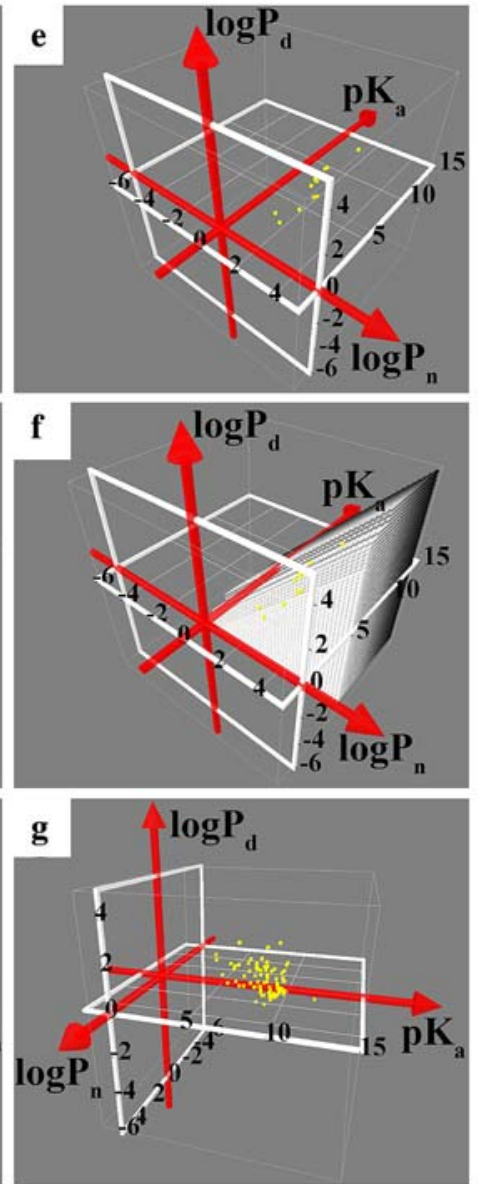
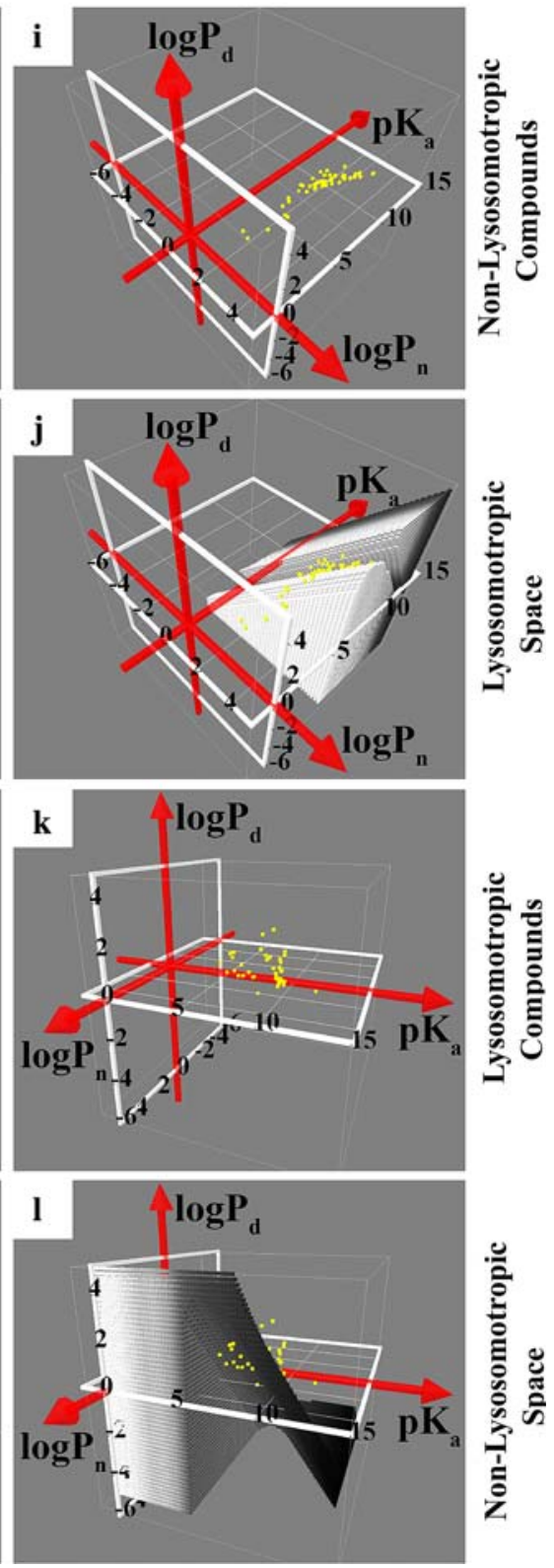
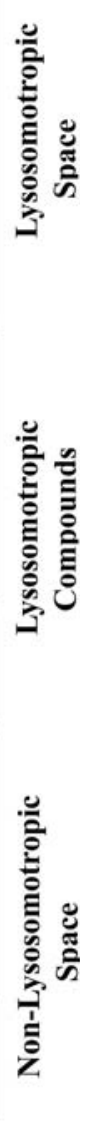

Fig. 2 Visualizing the simulated physicochemical property space occupied by lysosomotropic monobasic amines. Individual molecules in the test set are indicated by yellow dots. To discriminate between lysosomotropic vs. non-lysosomotropic molecules, three lysosomal concentrations were explored as thresholds: $2 \mathrm{mM}(\mathbf{a}-\mathbf{d}) ; 4 \mathrm{mM}(\mathbf{e}-\mathbf{h})$;

were below the accumulation threshold (Fig. 2e, f), while eighty-seven (87) were above the threshold (Fig. 2g, h). For compounds with a $\geq 8 \mathrm{mM}$ accumulation in lysosomes (Fig. 2i-1), fifty-six (56) lie below the accumulation threshold (Fig. 2i, j) while forty-three (43) are above the threshold (Fig. 2k, 1).

We established that a lysosomal accumulation threshold of $2 \mathrm{mM}$ is the best suited to distinguish lysosomotropic and $8 \mathrm{mM}(\mathbf{i}-\mathbf{l})$. Rows show non-lysosomotropic molecules (a, e, i); non-lysosomotropic molecules plus lysosomotropic space $(\mathbf{b}, \mathbf{f}, \mathbf{j})$; lysosomotropic molecules (c, g, k); and lysosomotropic molecules plus non-lysosomotropic space $(\mathbf{d}, \mathbf{h}, \mathbf{l})$

from non-lysosomotropic molecules, since it gave the most correct classification in terms of matching simulation results with the experimentally-observed, lysosomotropic behaviors. Accordingly, for a lysosomal accumulation threshold of $2 \mathrm{mM}$, of the 8 molecules that were below the accumulation threshold, $5(62.5 \%)$ have been positively identified as non-lysosomotropic. Conversely, of the 91 above the threshold, 8 (8.8\%) non-lysosomotropic 
molecules have been incorrectely classified as lysosomotropic. For a lysosomal accumulation threshold of $4 \mathrm{mM}$, of the 12 below the threshold, $5(41.7 \%)$ have been identified as non-lysosomotropic. Conversely, of the 87 above threshold, 8 (9.2\%) non-lysosomotropic molecules have been incorrectely classified as lysosomotropic. For a lysosomal accumulation threshold of $8 \mathrm{mM}$, of the 56 below the threshold, 9 have been positively identified as nonlysosomotropic (16.1\%). Conversely, of the 43 above the threshold, 4 (9.3\%) non-lysosomotropic molecules have been incorrectly classified as lysosomotropic.

The test set appears highly clustered in relation to the available lysosomotropic, physicochemical property space

Exploring the relationship between the physicochemical properties of the test set of molecules obtained from the literature with that of the theoretical physicochemical property space occupied by molecules that accumulate in lysosomes at the different threshold values, we observed that most of the test molecules tend to be clustered in very specific region of "lysosomotropic space". In fact, physicochemical property space occupied by molecules that accumulate in lysosomes at $\geq 2 \mathrm{mM}$ (Fig. 2b) appears largely similar to the space of molecules that accumulate at $\geq 4 \mathrm{mM}$ (Fig. 2f) and at $\geq 8 \mathrm{mM}$ (Fig. 2j). It was surprising that most lysosomotropic molecules in the reference set were calculated to have a lysosomal accumulation between 2- and 8-fold over the extracellular medium, although the largest portion of the calculated physicochemical property space that can be occupied by monobasic amines corresponds to $>8$-fold lysosomal accumulation.

Using simulation results to define the expected transport classes for monovalent weak bases

Using a 2-fold or greater concentration of drug over the extracellular medium to distinguish high vs. low lysosomal, mitochondrial and cytosolic concentration, and by incorporating high vs. low permeability classification obtained with the R-model, a total of 16 classes of molecules can be defined a priori (Table 1). By mapping the test set of molecules to these 16 different classes, we find that some classes of molecules are well-represented by a number of molecules, while other classes of molecules are not represented at all (Table 1). However, according to the simulation results, several of these a priori classifications are deemed to be "non-existent" by virtue of our being unable to find a combination of physicochemical properties consistent with the corresponding class of molecules in simulations.
Simulation results point to general trends in lysosomotropic behaviors

For the test set of molecules, we observed that the simulated intracellular accumulation in non-target cells (R-Model) is much lower than the corresponding accumulation in target cells (T-model) (Table 1). The simulations yielded lysosomal accumulation occurring for a broad range of transcellular permeability values (Table 1). Unexpectedly, for most lysosomotropic molecules, the simulations indicate that mitochondrial accumulation may be much greater than lysosomal or cytosolic accumulation, suggesting that lysosomotropic behavior may not be exclusively related to selective accumulation in lysosomes. Lastly, we observed that none of the lysosomotropic molecules in the test set are able to accumulate in cytosol to a greater extent than they accumulate in mitochondria or in lysosomes (Table 1). In fact, plotting the physicochemical property space of such molecules yielded an empty space (data not shown), indicating that the lack of such type of molecules in the reference set is not because the test set is a biased sample, but rather it is expected based on the calculated cellular pharmacokinetic properties of monovalent weak bases.

Calculating the physicochemical space occupied by selectively lysosomotropic molecules

Selectively lysosomotropic molecules were defined as those that accumulate in lysosomes to a 2-fold (or greater) level over the extracellular medium, cytosol, and mitochondria. Out of the 91 reference lysosomotropic molecules (Fig. 3a, out of circle), only seventeen (17) (Fig. 3c, d green circle) appear to be selective in terms of lysosomal accumulation. These 17 molecules (Fig. 3c) appear clustered at the middle $\mathrm{p} K_{\mathrm{a}}$ value of the test set of molecules comparing with non-lysosomotropic molecules (Fig. 3a, in blue circle) and non-selectively lysosomotropic molecules. Plotting the theoretical physicochemical property space occupied by selectively lysosomotropic molecules related to the reference molecules reveals that the test set of molecules that accumulate in lysosomes are highly clustered (Fig. 3b) in the middle $\mathrm{p} K_{\mathrm{a}}$ and high $\log P_{\mathrm{d}}$ values. This can also be observed in the corresponding plot of non-selectively lysosomotropic and non-lysosomotropic physicochemical property space (Fig. 3d).

Analyzing the effect of transcellular permeability on selective lysosomal accumulation

Next, we analyzed the relationship between selective lysosomal accumulation in target cells, and transcellular permeability in non-target cells, to determine if the ability to develop selective lysosomotropic agents may 
be constrained by desirably high transcellular permeability characteristics important for intestinal drug absorption and systemic tissue penetration (Fig. 4). As a reference, the permeability of metoprolol $\left(\mathrm{P}_{\mathrm{eff}}=35 \times\right.$ $10^{-6} \mathrm{~cm} / \mathrm{s}$ ) was used to distinguish high permeability from low permeability drugs. Accordingly, three permeability categories were defined: Negligible Permeability $\left(\mathrm{P}_{\text {eff }}<1 \times 10^{-6} \mathrm{~cm} / \mathrm{s} ; \quad\right.$ Fig. $4 \mathrm{a}, \quad$ b); Low Permeability $\left(1 \leq \mathrm{P}_{\text {eff }}<35 \times 10^{-6} \mathrm{~cm} / \mathrm{s}\right.$; Fig. $4 \mathrm{c}$, d $)$; and High Permeability $\left(\mathrm{P}_{\text {eff }} \geq 35 \times 10^{-6} \mathrm{~cm} / \mathrm{s}\right.$, Fig. $4 \mathrm{e}$, f).

With increasing permeability, the simulation results indicate that physicochemical space occupied by selective lysosomotropic molecules shifts towards lower $\mathrm{p} K_{\mathrm{a}}$ values and higher $\log P_{\mathrm{d}}$ values. The position of selective lysosomotropic chemical space in relation to the reference set of non-selective lysosomotropic or non- lysosomotropic molecules can be seen, for molecules with $\mathrm{P}_{\text {eff }}<1 \times 10^{-6}$ $\mathrm{cm} / \mathrm{s}$ (Fig. 4a); $1 \leq \mathrm{P}_{\text {eff }}<35 \times 10^{-6} \mathrm{~cm} / \mathrm{s}$ (Fig. 4c); and $P_{\text {eff }} \geq 35 \times 10^{-6} \mathrm{~cm} / \mathrm{s}$ (Fig. 4e). Accordingly, there is only one (1) selectively-lysosomotropic reference molecule with $\mathrm{P}_{\text {eff }}<1 \times 10^{-6} \mathrm{~cm} / \mathrm{sec}$ (Fig. 4b; green arrow); five (5) with $1 \leq$ Peff $<35 \times 10^{-6} \mathrm{~cm} / \mathrm{s}$ (Fig. $4 \mathrm{~d}$; green arrow); and eleven (11) with $\mathrm{P}_{\text {eff }} \geq 35 \times 10^{-6} \mathrm{~cm} / \mathrm{s}$ (Fig. 4f; green arrow). Thus, high permeability and selective lysosomal accumulation are not mutually exclusive. Nevertheless, we observed that the selective lysosomotropic reference molecules with negligibly low and high permeability are tightly clustered in a small region of chemical space, at mid $\mathrm{p} K_{\mathrm{a}}$ and high $\log P_{\mathrm{d}}$ values.

Demarcating the physicochemical property space of extracellular targeted molecules

Extracellular-targeted molecules can be defined as those whose intracellular accumulation at steady state is less than the extracellular concentration [24]. For drug development, such a class of molecules is important as many drug targets are extracellular. Accordingly, we analyzed simulation results to determine if there were molecules with low intracellular accumulation and high permeability, which would be desirable for the pharmaceutical design of orally absorbed drugs (Fig. 5). By maximizing permeability and minimizing intracellular accumulation, (using $\mathrm{P}_{\text {eff }} \geq 35 \times$ $10^{-6} \mathrm{~cm} / \mathrm{s}, \mathrm{C}_{\text {cyto }}<1 \mathrm{mM}, \mathrm{C}_{\text {mito }}<1 \mathrm{mM}$, and $\mathrm{C}_{\text {lyso }}<1$ $\mathrm{mM}$ as thresholds in both the $\mathrm{R}$ and $\mathrm{T}$ models), we found five (5) molecules falling into this class (Fig. 5a, b, c; green circle): pyrimidine, benzocaine, $\beta$-naphthylamine, 8 -aminoquinoline, and the anti-epileptic drug candidate AFCX1325XX. These are monobasic amines with $\mathrm{p} K_{\mathrm{a}}<4.5$. Molecules with $\mathrm{p} K_{\mathrm{a}}>4.5$ (the physicochemical property space shown in Fig. 5c) exhibit intracellular accumulation in lysosomes, cytosol or mitochondria to levels above those found in the extracellular medium. Figure $5 \mathrm{~b}$ shows the
Fig. 3 Visualizing the simulated physicochemical property space occupied by selectively lysosomotropic monobasic amines. Individual molecules in the test set are indicated by yellow dots. The four graphs show: (a) non-lysosomotropic molecules (inside blue circle) and non-selective lysosomotropic molecules (outside blue circle); (b) physicochemical property space occupied by selectively lysosomotropic molecules, in relation to non-lysosomotropic molecules (inside blue circle) and non-selective lysosomotropic molecules (outside blue circle); (c) selectively lysosomotropic molecules (inside green circle); (d) selectively lysosomotropic molecules (yellow dots in green circle) in relation to the union of non-selective lysosomotropic and non-lysosomotropic physicochemical property space
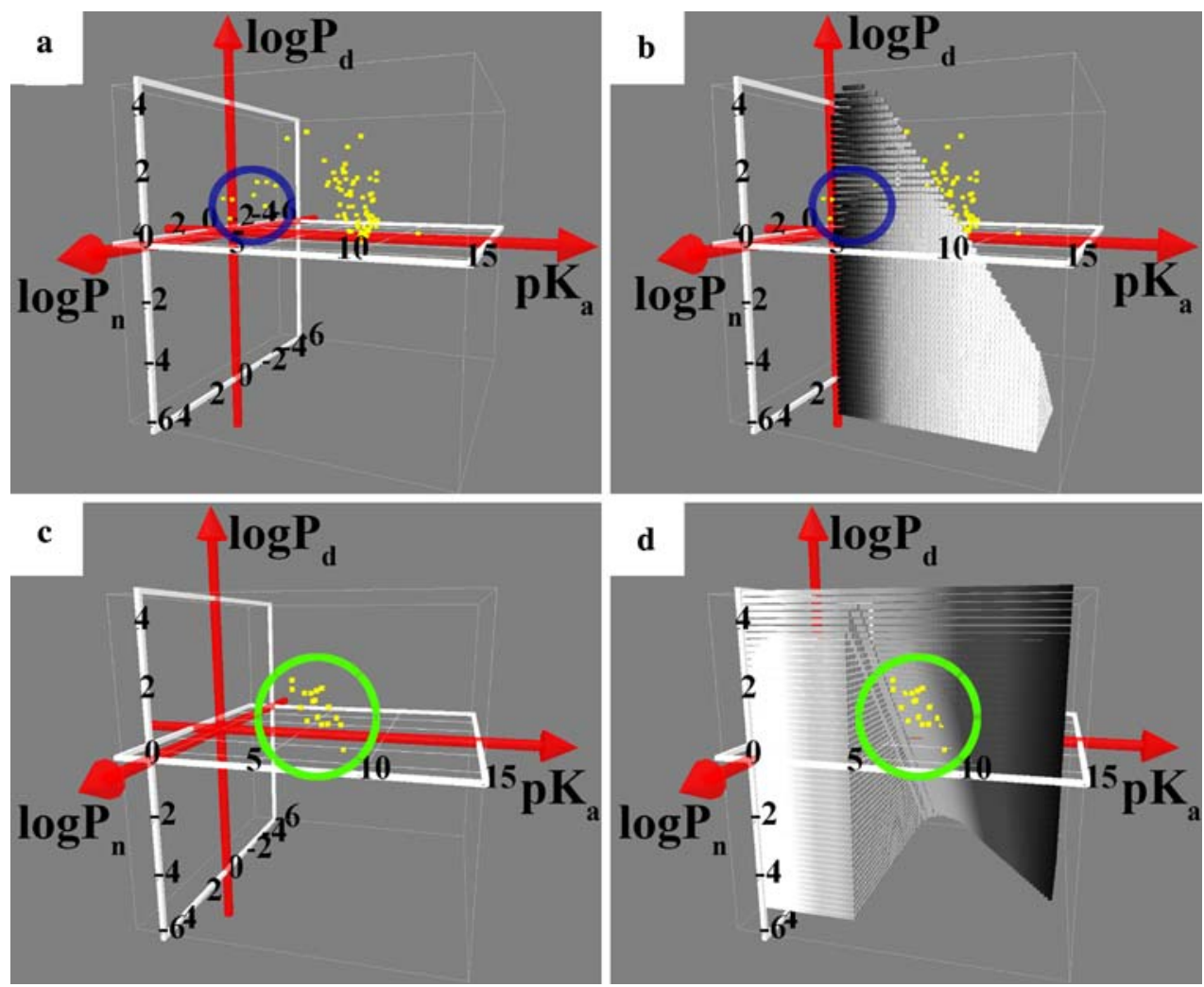

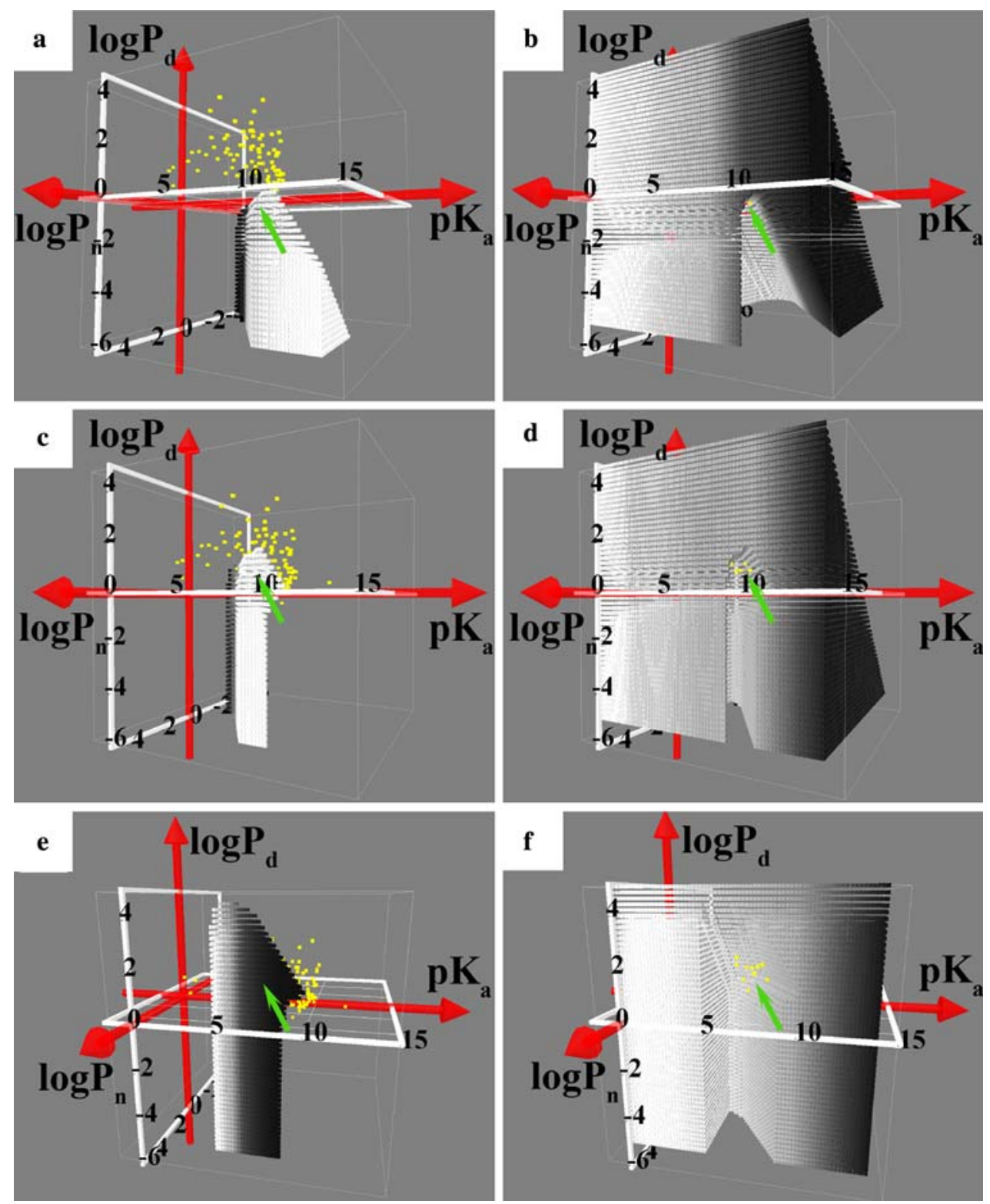

Fig. 4 Visualizing the relationship between transcellular permeability and lysosomotropic character. Individual molecules in the test set are indicated by yellow dots. The six graphs show: (a) physicochemical property space occupied by selectively lysosomotropic molecules with $\mathrm{P}_{\mathrm{eff}}<1 \times 10^{-6} \mathrm{~cm} / \mathrm{s}$, in relation to non-selectively lysosomotropic molecules, non-lysosomotropic molecules, and selectively lysosomotropic molecules with $\mathrm{P}_{\mathrm{eff}} \geq 1 \times 10^{-6} \mathrm{~cm} / \mathrm{s}$; (b) selectively lysosomotropic molecules with $\mathrm{P}_{\text {eff }}<1 \times 10^{-6} \mathrm{~cm} / \mathrm{s}$ (yellow dots) in relation to the union of physicochemical property spaces occupied by non-selectively lysosomotropic, non-lysosomotropic, and selectively lysosomotropic molecules with $\mathrm{P}_{\text {eff }} \geq 1 \times 10^{-6} \mathrm{~cm} / \mathrm{s}$; (c) physicochemical property space occupied by selectively lysosomotropic molecules with $1 \times 10^{-6} \mathrm{~cm} / \mathrm{s} \leq \mathrm{P}_{\text {eff }}<35 \times 10^{-6} \mathrm{~cm} / \mathrm{s}$, in relation to non-selectively lysosomotropic molecules, non-lysosomotropic molecules, and selectively lysosomotropic molecules with $\mathrm{P}_{\text {eff }}<$ $1 \times 10^{-6} \mathrm{~cm} / \mathrm{s}$ or $\mathrm{P}_{\text {eff }} \geq 35 \times 10^{-6} \mathrm{~cm} / \mathrm{s} ; \quad$ (d) selectively lysosomotropic molecules with $1 \times 10^{-6} \mathrm{~cm} / \mathrm{s} \leq \mathrm{P}_{\text {eff }}<35 \times 10^{-6}$ $\mathrm{cm} / \mathrm{s}$ in relation to the union of physicochemical property spaces occupied by non-selectively lysosomotropic molecules, non-lysosomotropic molecules, and selectively lysosomotropic molecules excluding those with $1 \times 10^{-6} \mathrm{~cm} / \mathrm{s} \leq \mathrm{P}_{\text {eff }}<35 \times 10^{-6} \mathrm{~cm} / \mathrm{s}$; $(\mathbf{e})$ physicochemical property space occupied by selectively lysosomotropic molecules with $\mathrm{P}_{\mathrm{eff}} \geq 35 \times 10^{-6} \mathrm{~cm} / \mathrm{s}$, in relation to nonselectively lysosomotropic molecules, non-lysosomotropic molecules and lysosomotropic molecules with $\mathrm{P}_{\text {eff }}<35 \times 10^{-6} \mathrm{~cm} / \mathrm{s}$; (f) selectively lysosomotropic molecules with $\mathrm{P}_{\mathrm{eff}} \geq 35 \times 10^{-6} \mathrm{~cm} / \mathrm{s}$ in relation to the union of physicochemical property spaces occupied by non-selectively lysosomotropic, non-lysosomotropic, and selectively lysosomotropic molecules with $\mathrm{P}_{\mathrm{eff}}<35 \times 10^{-6} \mathrm{~cm} / \mathrm{s}$. Green arrow point to the general region of physicochemical property space where the reference molecules are visibly clustered 

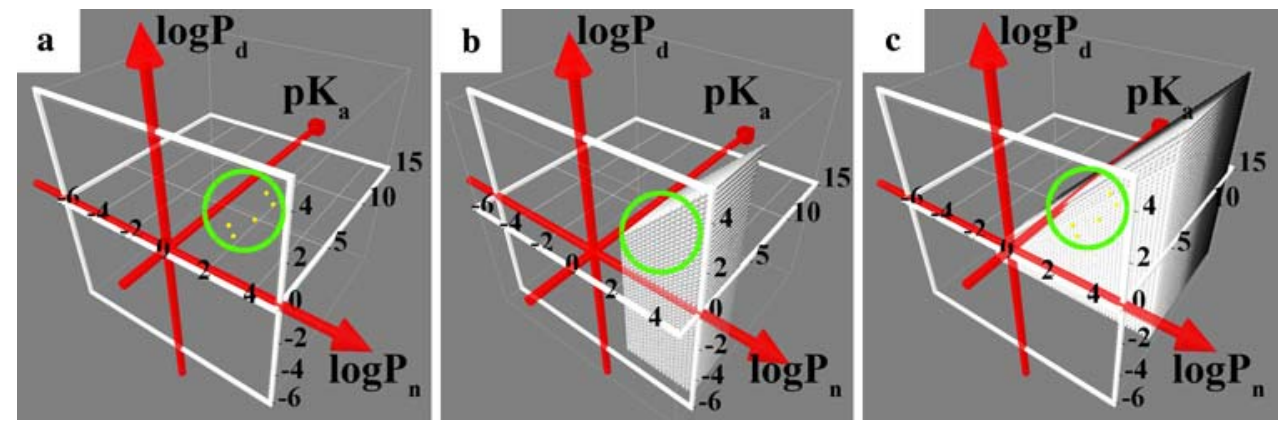

Fig. 5 Visualizing the simulated physicochemical property space occupied by molecules with low intracellular accumulation and high permeability. Individual molecules in the test set are indicated by yellow dots. The three graphs show: (a) molecules with low intracellular accumulation and high permeability (inside green circle);

physicochemical space of molecules with high permeability and low intracellular accumulation. Figure $5 \mathrm{c}$ shows the physicochemical space of molecules with high intracellular accumulation regardless of permeability. Again we can see that molecules with low intracellular accumulation have a $\mathrm{p} K_{\mathrm{a}}<4.5$ and with high intracellular accumulation have a $\mathrm{p} K_{\mathrm{a}}>4.5$.

Many reported lysosomotropic molecules appear to accumulate in mitochondria

For the majority of the reportedly lysosomotropic monobasic amines in the test set, the model suggests that they accumulate in mitochondria more than they accumulate in lysosomes. In total, 56 of the 91 lysosomotropic molecules in the test set accumulate in mitochondria at 2-fold or greater levels than they accumulate in lysosomes, cytosol, or the extracellular medium (Fig. 6a; Table 1, selectively mitochondrotropic compounds underlined). These molecules have a $\mathrm{p} K_{\mathrm{a}}$ of 8.2 or greater, a $\log P_{\mathrm{n}}$ of 1.5 or greater, and span a wide range of transcellular permeability values-from impermeant to very highly permeant. In addition, eighteen (18) lysosomotropic molecules also exhibit mitochondrial and high cytosolic accumulation, at concentrations comparable to the concentrations at which they accumulate in lysosomes (Fig. 6b; Table 1). Again, these molecules span a broad range of transcellular permeability values, from impermeant to highly permeant. Plotting the theoretical physicochemical property space occupied by lysosomotropic molecules with predicted, selective mitochondrial accumulation reveals that the molecules in the test set are clustered in this realm of physicochemical property space (Fig. 6c). Similarly, plotting the physicochemical property space occupied by lysosomotropic molecules that are predicted to accumulate in cytosol and mitochondria reveals that the molecules are clustered in this realm of chemical space. (b) physicochemical property space occupied by molecules with calculated low intracellular accumulation and high permeability (green circle same as in a); (c) the simulated physicochemical property space occupied by molecules with high intracellular accumulation, regardless of permeability (green circle same as in a)

Calculated effect of $\mathrm{pH}$ in apical compartment on permeability and biodistribution

Based on the simulations, the accumulation of monobasic amines in lysosomes is largely dependent on the difference in $\mathrm{pH}$ of between lysosome and extracellular medium (data not shown). While the $\mathrm{pH}$ of the medium bathing the target cells is expected to be rather constant, the $\mathrm{pH}$ surrounding an intestinal epithelial cell is expected to vary along the intestinal tract [45]. To test if this variation would lead to major differences in the observed trends, we decided to test the extent to which the calculated chemical space occupied by selectively lysosomotropic molecules was affected by variation in the apical pH of non-target cells (Fig. 7). We note that for selectively lysosomotropic molecules with negligible (Fig. 7a), low (Fig. 7b), and high (Fig. 7c) permeability, the theoretical physicochemical property space occupied by selectively lysosomotropic molecules is similar, and the test molecules that fall into that region of chemical space tend to be the same. Similarly, other regions of physicochemical property space occupied with molecules of different permeability tend to be similar, with variations in the apical $\mathrm{pH}$ of the intestinal epithelial cell in a pH range of 4.5-6.8 (data not shown).

\section{Discussion}

Modeling the cellular pharmacokinetics of monobasic amines

Over the past few years, mathematical models of cellular pharmacokinetics have been developed, based on coupled sets of differential equations capturing the transmembrane diffusion of small molecules. Previously, these models have been used to simulate the intracellular distribution of lipophilic cations in tumor cells [25], and the distribution 
Fig. 6 Visualizing the simulated physicochemical property space of various classes of non-selective, lysosomotropic molecules. Individual molecules in the test set are indicated by yellow dots. The four graphs show: (a) fifty-six selectively mitochondriotropic molecules; (b) 18 lysosomotropic, molecules which are not selective in terms of lysosomal, mitochondrial or cytosolic accumulation; (c) the simulated physicochemical property space occupied by lysosomotropic molecules that are also selectively mitochondriotropic; (d) the simulated physicochemical property space of non-selective lysosomotropic, non-selective mitochondriotropic molecules
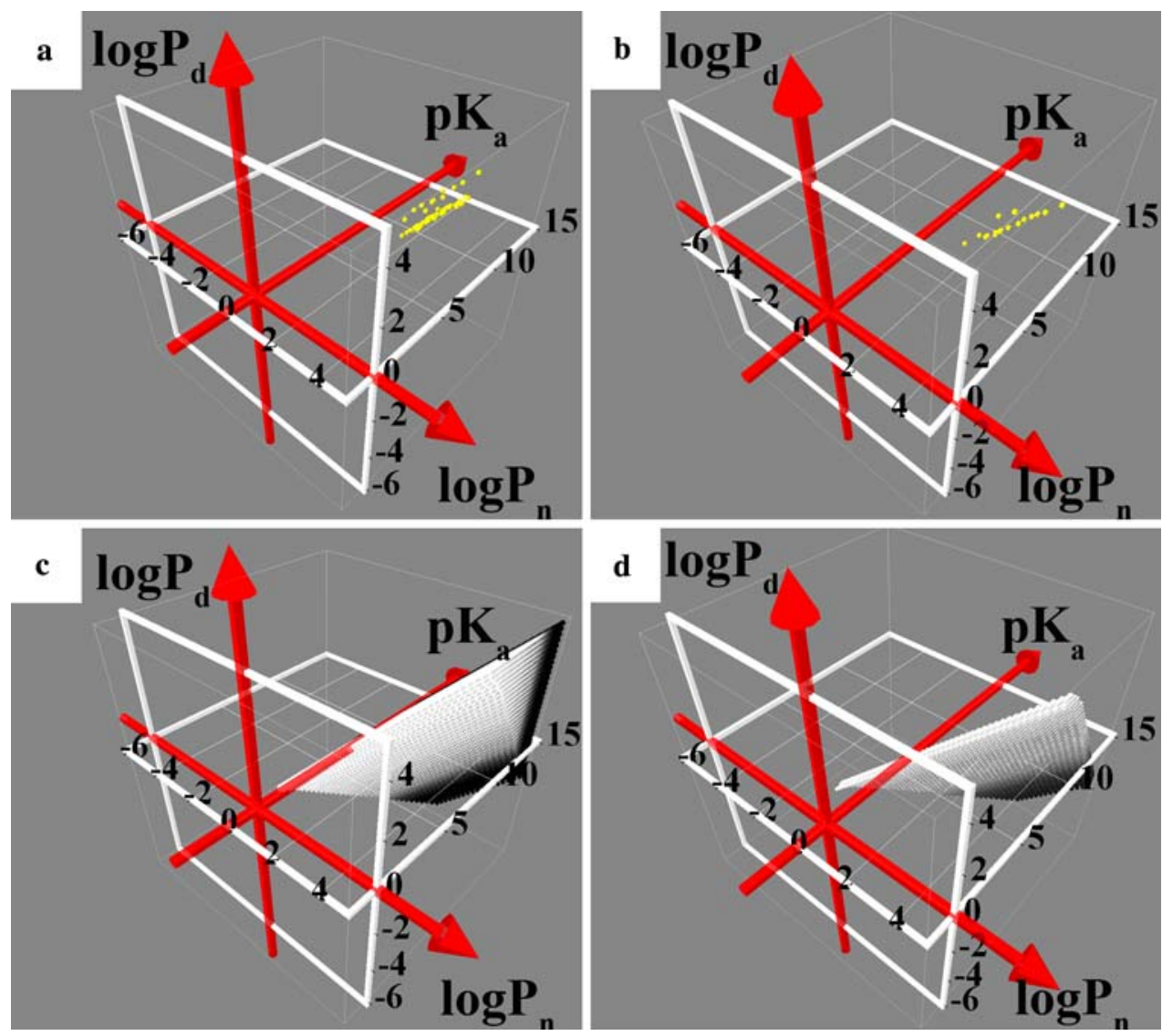

and transport of small molecules across intestinal epithelial cells [24]. For a monovalent weakly acidic or weakly basic small molecule drug, three input physical-chemical properties are used to simulate cellular drug transport and distribution: the logarithms of the lipid/water partition coefficient of the neutral form of the molecule $\left(\log P_{\mathrm{n}}\right)$ and ionized form $\left(\log P_{\mathrm{d}}\right)$, and the negative $\operatorname{logarithm}$ of the dissociation constant of the ionizable group $\left(\mathrm{p} K_{\mathrm{a}}\right)$. For monovalent weak bases, the transcellular permeability values calculated with this approach were comparable with measured human intestinal permeability and Caco-2 permeability, yielding good predictions [24]. Similarly, the corresponding mathematical models were able to predict mitochondrial accumulation of lipophilic cationic substances in tumor cells [22, 25].

For analyzing the lysosomotropic behavior of monovalent weak bases possessing amine functionality, we adapted these two mathematical models to simulate the cellular pharmacokinetic behavior of target cells exposed to a homogeneous extracellular drug concentration, and nontarget cells mediating drug absorption in the presence of an apical-to-basolateral concentration gradient. The results we obtained establish a baseline, expected concentration of small drug-like molecules in mitochondria, lysosomes and cytosol of target cells, as well as permeability in non-target cells. With a test set of small molecules obtained from published research articles, the simulations permit exploring the relationship between physicochemical properties of the molecules, their simulated intracellular distributions and transport behavior, and experimentally reported cellular phenotypes.

Simulation-based analysis and classification of lysosomotropic behavior

By analyzing the intracellular distribution and transcellular transport characteristics of a test set of molecules, together with more general physicochemical space plots covering all possible combinations of $\mathrm{p} K_{\mathrm{a}}, \log P_{\mathrm{n}}$ and $\log P_{\mathrm{d}}$, sixteen a priori classes of lysosomotropic behavior for monobasic amines were defined (Table 1). However, we noted that several of these classes are deemed to be non-existent by the simulations - meaning that there is no combination of $\mathrm{p} K_{\mathrm{a}}, \log P_{\mathrm{n}}$ and $\log P_{\mathrm{d}}$ that will yield a molecule in such a class. For other classes, it was not possible to find a molecule in the reference set of lysosomotropic molecules whose calculated properties would lie within the physicochemical property space defining the hypothetical class of molecules. This is certainly the case for positivelyidentified, non-lysosomotropic molecules. These results 
Fig. 7 Visualizing the effect of extracellular $\mathrm{pH}$ on physicochemical property space occupied by selectivelylysosomotropic molecules. Simulations were carried out using an apical $\mathrm{pH}$ of $4.5(\mathbf{a}-\mathbf{c})$ and $6.8(\mathbf{d}-\mathbf{f})$ in the R-Model. Yellow dots indicate individual molecules in the test set. Each row shows the physicochemical property space occupied by molecules in different permeability classes, as follows:

(a) and (d) $\mathrm{P}_{\text {eff }}<1 \times 10^{-6} \mathrm{~cm} / \mathrm{s}$;

(b) and (e) $1 \times 10^{-6} \mathrm{~cm} / \mathrm{s} \leq$

$\mathrm{P}_{\text {eff }}<35 \times 10^{-6} \mathrm{~cm} / \mathrm{s}$,

(d) and (f) $\mathrm{P}_{\text {eff }} \geq 35 \times$

$10^{-6} \mathrm{~cm} / \mathrm{s}$
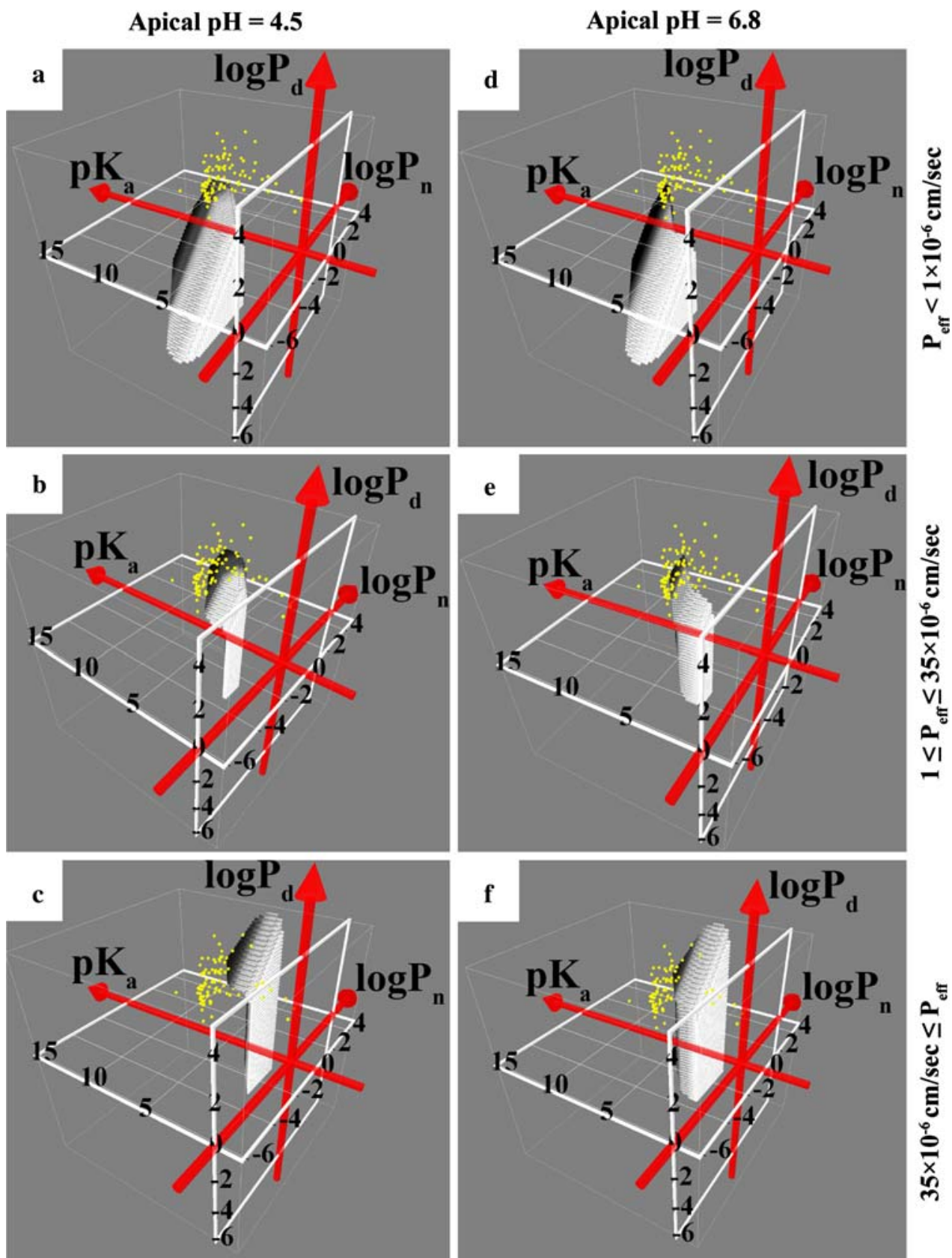

argue for expanding the test set of monovalent, weakly basic molecules, so as to represent all possible classes of intracellular transport behaviors.

An equally important observation from the simulation resides in the tight clustering of the reference molecules in constrained regions of physicochemical property space, in relation to the simulated physicochemical property space that is actually available for molecules in the different lysosomotropic and permeability categories. Thus, the diversity of lysosomotropic behaviors represented by the test set of molecules is significantly limited. Indeed, the simulations indicate that expanding the reference set of molecules to unexplored regions of physicochemical property space could be used to find molecules that better represent different types of expected cellular pharmacokinetic behaviors. For example, in the case of low or high permeability molecules that are selectively lysosomotropic, most of the molecules in the reference set are clustered at the high levels of $\mathrm{p} K_{\mathrm{a}}$ and high $\log P$, whereas the simulations indicate that it should be possible to find molecules with lower $\mathrm{p} K_{\mathrm{a}}$ and $\operatorname{lower} \log P$. The reason for the limited chemical diversity of reported lysosomotropic molecules is certainlly related to the choice of molecules that have been tested experimentally and reported in the literature: the 
emphasis has not been on the probing the chemical diversity of lysosomotropic character, but rather, in analyzing the lysosomotropic character in a related series of compounds (for example, studies looking at mono, bi, and trisubstituted amines, functionalized with various aliphatic groups [9]). In other cases, the emphasis has been on studying the lysosomotropic character of a specific type of compound developed against a specific drug target [6] (for example, beta-adrenergic receptor antagonists such as propranolol, atenolol, practolol, etc), rather than on the full chemical space occupied by lysosomotropic, monovalent weakly basic amines.

Further experimental validation and testing of expected transport behaviors

Using lysosomal swelling, cell vacuolation and intralysosomal $\mathrm{pH}$ measurements as phenotypic read outs, it may be possible to test both $\mathrm{R}$ - and $\mathrm{T}$-model prediction about the varying extent of lysosomal accumulation of monovalent weak bases as a function of the molecule's chemical structure or physicochemical properties. For example, the models make quantitative predictions about the lysosomal concentration of molecules of varying chemical structure. Previous studies looking at the lysosomotropic behavior of various molecules have reported differences in vacuolation induction for different probes, at extracellular drug concentrations ranging from high millimolar to micromolar range $[10,13,16]$. Also, for some molecules vacuolation occurs after less than an hour incubation, while for other probes vacuolation occurs after twenty-four hour incubation, or longer $[6,9,10,13,14,16]$. Combinatorial libraries of fluorescent molecules are available today [46, 47], offering yet another way to test predictions about the intracellular accumulation and distribution of probes. Furthermore, with organelle-selective markers and kinetic microscopic imaging instruments, the rate and extent of swelling of lysosomes and other organelles could be monitored dynamically after exposure of cells to monovalent weakly basic molecules [47]. For such studies, cheminformatic analysis tools are being developed to relate the intracellular distribution of small molecules as apparent in image data, with chemical structure and physicochemical features of the molecules, and the predicted subcellular distribution [48, 49]. Lastly, more quantitative assessments of model predictions can be made by directly monitoring the total intracellular drug mass [50,51], as well as drug mass associated with the lysosomal compartment [20, 52, 53]. Recently, methods are being developed to rapidly isolate the lysosomes and measure intralysosomal drug concentrations [53].

To test model predictions about the lysosomotropic behavior of small molecules in the presence of an apical-to-basolateral concentration gradient, various in vitro cell culture models have been developed to assess drug intestinal permeability and oral absorption [54]. These are Caco-2, MDCK, LLC-PK1, 2/4/A1, TC-7, HT-29, and IEC-18 cell models [54]. Among those models Caco-2 (human colon adenocarcinoma) cell monolayer is the most well-established cell model and has been widely accepted by pharmaceutical companies and academic research groups interested in studying drug permeability characteristics [54]. In addition to Caco-2 cells, MDCK (MadinDarby canine kidney) is a dog-renal epithelia cell line and is another widely used cell line in studying cell permeability characteristics [55].

Towards a computer-aided design of organelle-targeted molecules: implications for drug discovery and development

The ability to rationally tailor the transcellular permeability and subcellular distribution of monobasic amines can have important applications in medicinal chemistry efforts aimed at enhancing the efficacy of small molecules against specific targets, decreasing non-specific unwanted interactions with non-intended targets that lead to side effects and toxicity, as well as enhancing transcellular permeability for maximizing tissue penetration and oral bioavailability. For many FDA approved drugs, lysosomal accumulation of the molecules would appear to be a non-specific effect of the molecule's chemical structure. For example, in the case of the beta-adrenergic receptor antagonists like propranolol, the drug's target is a cell surface receptor located at the plasma membrane. Thus, lysosomal (and any other intracellular) accumulation observed for this molecule is most likely an unintended consequence of its chemical structure $[2,6,15,16,41,53]$. In general, due to the abundance of lysosomotropic drugs $[6,9,10,16]$, lysosomal accumulation seems to be tolerated, although it may not be a desirable property.

Nevertheless, there are certain classes of therapeutic agents where lysosomal accumulation may be highly desirable. For example, Toll-like receptor molecules are transmembrane proteins in the lysosomes of leukocytes (dendritic cells and macrophages). These receptors can be activated by endocytosed proteins, DNA and carbohydrates, and they generate inflammatory responses as part of the innate immune system [56, 57]. Small molecule agents that either block or activate Toll-like receptors are being sought to inhibit inflammatory reactions (associated with autoimmune diseases) or promote resistance against viral infections, respectively $[58,59]$. A different class of molecules where lysosomal accumulation would be highly desirable involves agents that affect lysosomal enzymes involved in tissue remodeling [60]. Tissue remodeling is 
the basis of diseases like osteoporosis, which involves the loss of bone mass due to an imbalance in the rate of bone deposition and bone resorption.

From the simulations, mitochondria also appear as an important site of accumulation of monobasic amineseven for many molecules that have been previously classified as being "lysosomotropic". Our simulation results indicate that monovalent weak bases can selectively accumulate in mitochondria at very high levels -in fact, at much higher levels than they appear to be able to accumulate in lysosomes. From a drug toxicity standpoint, unintended accumulation of small molecules in mitochondria can interfere with mitochondrial function, leading to cellular apoptosis [61-63]. Conversely, intentional targeting of small molecule therapeutic agents to mitochondria can be a desirable feature for certain classes of drugs: mitochondria dysfunction can cause a variety of diseases, so there is great interest in developing mitochondriotropic drugs [22, 64-66].

Nevertheless, perhaps the most important classes of subcellularly-targeted molecules are those that are aimed at extracellular domains of cell surface receptors [24]. Many 'blockbuster' drugs in the market today target cell surface receptors, ion channels, and other extracellular enzymes, making extracellular space one of the most valuable sitesof-action for drug development [67]. Extracellular-acting therapeutic agents include anticoagulants that interfere with clotting factors in the blood, agents that interfere with pro-hormone processing enzymes, ion channel blockers for treating heart conditions, GPCR antagonists for hypertension, inflammation and a variety of other different conditions, and many CNS-active agents that act on neurotransmitter receptors, transport and processing pathways. In order to target extracellular domains of blood proteins, cell surface receptors and ion channels, it is desirable that a molecule would have high transcellular permeability to facilitate absorption and tissue penetration. In addition, it would be desirable that the molecule would also have low intracellular accumulation so as to maximize extracellular concentration. The simulation results indicate that indeed, finding monovalent weak bases with high permeability and low intracellular accumulation in both target and non-target cells is possible, with several molecules in the reference set residing in this realm of physicochemical property space.

To conclude, cell based molecular transport simulators constitute a promising cheminformatic analysis tool for analyzing the subcellular transport properties of small molecules. The ability to combine results from different models, visualize simulations representing hundreds of thousands of different combinations of physicochemical properties, and relate these simulation results to the chemical structure and phenotypic effects of specific drugs and small drug-like molecules adds a new dimension to the existing mathematical models. As related to the specific class of lysosomotropic monobasic amines analyzed in this study, interactive visualization of simulation results point to a richness in subcellular transport and distribution behavior that is otherwise difficult to appreciate. We anticipate that the complexity of subcellular transport behaviors will ultimately be exploited in future generations of small molecule drug candidates "supertargeted" to their sites of action [68], be it in the extracellular space, the cytosol, mitochondria, lysosomes and potentially other intracellular organelles.

Acknowledgements The authors thank Kerby Shedden and Vivien Chen for critical reading of the manuscript. This work was funded by NIH grants RO1GM078200, P20HG003890 and R21CA104686 to G.R.R.

Open Access This article is distributed under the terms of the Creative Commons Attribution Noncommercial License which permits any noncommercial use, distribution, and reproduction in any medium, provided the original author(s) and source are credited.

\section{References}

1. Kaufmann AM, Krise JP (2007) J Pharm Sci 96:729-746

2. Duvvuri M, Gong YP, Chatterji D, Krise JP (2004) J Biol Chem 279:32367-32372

3. de Duve C, de Barsy T, Poole B, Trouet A, Tulkens P, Van Hoof F (1974) Biochem Pharmacol 23:2495-2531

4. Reasor MJ, Kacew S (2001) Exp Biol Med (Maywood) 226: $825-830$

5. Fujimura H, Dekura E, Kurabe M, Shimazu N, Koitabashi M, Toriumi W (2007) Exp Toxicol Pathol 58:375-382

6. Cramb G (1986) Biochem Pharmacol 35:1365-1372

7. Styrt B, Klempner MS (1988) Biochem Pharmacol 37:435-441

8. Ploemen JP, Kelder J, Hafmans T, van de Sandt H, van Burgsteden JA, Saleminki PJ, van Esch E (2004) Exp Toxicol Pathol 55:347-355

9. Seglen PO, Gordon PB (1980) Mol Pharmacol 18:468-475

10. Ohkuma S, Poole B (1981) J Cell Biol 90:656-664

11. Honegger UE, Quack G, Wiesmann UN (1993) Pharmacol Toxicol 73:202-208

12. Honegger UE, Roscher AA, Wiesmann UN (1983) J Pharmacol Exp Ther 225:436-441

13. Morissette G, Moreau E, C-Gaudreault R, Marceau F (2004) J Pharmacol Exp Ther 310:395-406

14. Morissette G, Moreau E, C-Gaudreault R, Marceau F (2005) Mol Pharmacol 68:1576-1589

15. Hurwitz SJ, Terashima M, Mizunuma N, Slapak CA (1997) Blood 89:3745-3754

16. Ishizaki J, Yokogawa K, Ichimura F, Ohkuma S (2000) J Pharmacol Exp Ther 294:1088-1098

17. Lemieux B, Percival MD, Falgueyret JP (2004) Anal Biochem 327:247-251

18. Yang WC, Strasser FF, Pomerat CM (1965) Exp Cell Res 38:495-506

19. Bulychev A, Trouet A, Tulkens P (1978) Exp Cell Res 115:343-355

20. Duvvuri M, Krise JP (2005) Mol Pharm 2:440-448

21. Cabantchik ZI, Silfen J, Firestone RA, Krugliak M, Nissani E, Ginsburg H (1989) Biochem Pharmacol 38:1271-1277 
22. Horobin RW, Trapp S, Weissig V (2007) J Control Release 121:125-136

23. Gonzalez-Rothi RJ, Zander DS, Ros PR (1995) Chest 107: $1763-1765$

24. Zhang X, Shedden K, Rosania GR (2006) Mol Pharm 3:704-716

25. Trapp S, Horobin RW (2005) Eur Biophys J Biophys Lett 34:959-966

26. Loew LM, Tuft RA, Carrington W, Fay FS (1993) Biophys J 65:2396-2407

27. Rybak SL, Murphy RF (1998) J Cell Physiol 176:216-222

28. Cain CC, Murphy RF (1988) J Cell Biol 106:269-277

29. Preston RA, Murphy RF, Jones EW (1989) Proc Natl Acad Sci USA 86:7027-7031

30. Van Dyke RW (1988) J Biol Chem 263:2603-2611

31. Felber SM, Brand MD (1982) Biochem J 204:577-585

32. Simon S, Roy D, Schindler M (1994) Proc Natl Acad Sci USA 91:1128-1132

33. Zhang X, Rosania GR (2006) A virtual cell-based simulator for pharmaceutical project management, risk assessment and decision making. Proceeding for the 4th International Conference on Computer Science and its Applications, San Diego, CA, USA, pp 58-65

34. Duvvuri M, Konkar S, Hong KH, Blagg BS, Krise JP (2006) ACS Chem Biol 1:309-315

35. Li W, Yuan XM, Ivanova S, Tracey KJ, Eaton JW, Brunk UT (2003) Biochem J 371(Pt2):429-436

36. Schneider P (1992) Arch Toxicol 66:23-33

37. Schmid BP, Hauser RE, Donatsch P (1985) Xenobiotica 15: 695-699

38. Daniel WA, Wojcikowski J, Palucha A (2001) Br J Pharmacol 134:807-814

39. Ishizaki J, Yokogawa K, Hirano M, Nakashima E, Sai Y, Ohkuma S, Ohshima T, Ichimura F (1996) Pharm Res 13:902-906

40. Drenckhahn D, Kleine L, Lullmann-Rauch R (1976) Lab Invest 35:116-123

41. McCloud CM, Beard TL, Kacew S, Reasor MJ (1995) Exp Mol Pathol 62:12-21

42. Hetman M, Danysz W, Kaczmarek L (1997) Exp Neurol 147:229-237

43. Pessayre D, Bichara M, Degott C, Potet F, Benhamou JP, Feldmann G (1979) Gastroenterology 76:170-177

44. Balon K, Riebesehl BU, Muller BW (1999) Pharm Res 16: 882-888

45. Charman WN, Porter CJ, Mithani S, Dressman JB (1997) J Pharm Sci 86:269-282

46. Rosania GR, Lee JW, Ding L, Yoon HS, Chang YT (2003) J Am Chem Soc 125:1130-1131
47. Chen VY, Khersonsky SM, Shedden K, Chang YT, Rosania GR (2004) Mol Pharm 1:414-425

48. Posada MM, Shedden K, Chang YT, Li Q, Rosania GR (2007) Live cell bioimaging probes with cheminformatic assisted image arrays (CAIA). Proceedings for the 4th IEEE International Symposium on Biomedical Imaging, Metro Washington, DC, USA, pp 1791-1802

49. Shedden K, Brumer J, Chang YT, Rosania GR (2003) J Chem Inf Comput Sci 43:2068-2080

50. Chen VY, Posada MM, Blazer LL, Zhao T, Rosania GR (2006) Pharm Res 23:1687-1695

51. Chen VY, Posada MM, Zhao L, Rosania GR (2007) Pharm Res 24:2156-2167

52. Duvvuri M, Feng W, Mathis A, Krise JP (2004) Pharm Res 21:26-32

53. Gong Y, Zhao Z, McConn DJ, Beaudet B, Tallman M, Speake JD, Ignar DM, Krise JP (2007) Pharm Res 24:1138-1144

54. Balimane PV, Chong S (2005) Drug Discov Today 10:335-343

55. Irvine JD, Takahashi L, Lockhart K, Cheong J, Tolan JW, Selick HE, Grove JR (1999) J Pharm Sci 88:28-33

56. Matsumoto M, Funami K, Tanabe M, Oshiumi H, Shingai M, Seto Y, Yamamoto A, Seya T (2003) J Immunol 171:3154-3162

57. Kawai T, Akira S (2007) Semin Immunol 19:24-32

58. Fletcher S, Steffy K, Averett D (2006) Curr Opin Investig Drugs 7:702-708

59. Cristofaro P, Opal SM (2006) Drugs 66:15-29

60. Falgueyret JP, Desmarais S, Oballa R, Black WC, Cromlish W, Khougaz K, Lamontagne S, Masse F, Riendeau D, Toulmond S, Percival MD (2005) J Med Chem 48:7535-7543

61. van Ginkel PR, Sareen D, Subramanian L, Walker Q, Darjatmoko SR, Lindstrom MJ, Kulkarni A, Albert DM, Polans AS (2007) Clin Cancer Res 13:5162-5169

62. Jacotot E, Deniaud A, Borgne-Sanchez A, Touat Z, Briand JP, Le Bras M, Brenner C (2006) Biochim Biophys Acta 1757: $1312-1323$

63. Fantin VR, Berardi MJ, Scorrano L, Korsmeyer SJ, Leder P (2002) Cancer Cell 2:29-42

64. D'Souza GG, Boddapati SV, Weissig V (2007) Pharm Res 24:228-238

65. Weissig V, Torchilin VP (2000) Curr Pharm Biotechnol 1: 325-346

66. D'Souza GG, Rammohan R, Cheng SM, Torchilin VP, Weissig V (2003) J Control Release 92:189-197

67. Overington JP, Al-Lazikani B, Hopkins AL (2006) Nat Rev Drug Discov 5:993-996

68. Rosania GR (2003) Curr Top Med Chem 3:659-685 